\title{
Progress towards the first measurement of charm baryon dipole moments
}

\author{
S. Aiola $\odot,{ }^{1}$ L. Bandiera $\odot,{ }^{2}$ G. Cavoto $\odot,{ }^{3,4}$ F. De Benedetti, ${ }^{1}$ J. Fu, ${ }^{1,5}$ V. Guidi, ${ }^{2,6}$ L. Henry, ${ }^{1,5,7}$ D. Marangotto, ${ }^{1,5}$ \\ F. Martinez Vidal, ${ }^{7}$ V. Mascagna $\odot,{ }^{8,9}$ J. Mazorra de Cos, ${ }^{7}$ A. Mazzolari, ${ }^{2}$ A. Merli, ${ }^{1,5}$ N. Neri $\odot,{ }^{1,5}$ M. Prest $\odot,{ }^{8,9}$ \\ M. Romagnoni, ${ }^{2,5}$ J. Ruiz Vidal $\odot,{ }^{7}$ M. Soldani,${ }^{8,9, *}$ A. Sytov, ${ }^{2}$ V. Tikhomirov $\odot,{ }^{10}$ and E. Vallazza ${ }^{8}$ \\ ${ }^{1}$ INFN Sezione di Milano, Milan, Italy \\ ${ }^{2}$ INFN Sezione di Ferrara, Ferrara, Italy \\ ${ }^{3}$ INFN Sezione di Roma, Rome, Italy \\ ${ }^{4}$ Università di Roma "La Sapienza”, Rome, Italy \\ ${ }^{5}$ Università degli Studi di Milano, Milan, Italy \\ ${ }^{6}$ Università degli Studi di Ferrara, Ferrara, Italy \\ ${ }^{7}$ IFIC, Universitat de València-CSIC, Valencia, Spain \\ ${ }^{8}$ INFN Sezione di Milano Bicocca, Milan, Italy \\ ${ }^{9}$ Università degli Studi dell'Insubria, Como, Italy \\ ${ }^{10}$ Institute for Nuclear Problems and Belarusian State University, Minsk, Belarus
}

(Received 26 November 2020; accepted 12 February 2021; published 12 April 2021)

\begin{abstract}
Electromagnetic dipole moments of short-lived particles are sensitive to physics within and beyond the Standard Model of particle physics but have not been accessible experimentally to date. To perform such measurements it has been proposed to exploit the spin precession of channeled particles in bent crystals at the LHC. Progress that enables the first measurement of charm baryon dipole moments is reported. In particular, the design and characterization on beam of silicon and germanium bent crystal prototypes, the optimization of the experimental setup, and advanced analysis techniques are discussed. Sensitivity studies show that first measurements of $\Lambda_{c}^{+}$and $\Xi_{c}^{+}$baryon dipole moments can be performed in two years of data taking with an experimental setup positioned upstream of the $\mathrm{LHCb}$ detector.
\end{abstract}

DOI: 10.1103/PhysRevD.103.072003

\section{INTRODUCTION}

Electromagnetic dipole moments are static properties of particles that are sensitive to physics within and beyond the Standard Model (SM) of particle physics. For particles like the proton, neutron, muon and electron, such measurements provide among the most stringent tests of the SM [1-7]. In classical physics, the magnetic dipole moment (MDM) measures the strength and orientation of the magnetic field generated by the motion of electric charges or by the intrinsic magnetism of matter. In particle physics, the MDM is proportional to the particle spin-polarization vector $\boldsymbol{s}$ and for spin-1/2 particles is given by $\boldsymbol{\mu}=$ $g \mu_{\mathrm{B}} s / 2$ (Gaussian units), where $g$ is the dimensionless gyromagnetic factor, $\mu_{\mathrm{B}}=e \hbar /(2 m c)$ is the particle magneton, and $m$ its mass. The spin polarization is a unit vector defined as $\boldsymbol{s}=2\langle\boldsymbol{S}\rangle / \hbar$, where $\boldsymbol{S}$ is the spin operator. The measurement of the baryon MDM provides experimental

\footnotetext{
*Present address: INFN Sezione di Ferrara, Ferrara, Italy.
}

Published by the American Physical Society under the terms of the Creative Commons Attribution 4.0 International license. Further distribution of this work must maintain attribution to the author(s) and the published article's title, journal citation, and DOI. Funded by SCOAP ${ }^{3}$. anchor points for low energy models of strong interactions, while for leptons can be confronted with precise calculations for a stringent SM test [8,9]. The electric dipole moment (EDM) of a system measures the separation of the positive and negative electric charge distribution. The particle EDM is proportional to its spin-polarization vector and is defined as $\boldsymbol{\delta}=d \mu_{\mathrm{B}} \boldsymbol{s} / 2$, where $d$ is the gyroelectric factor. The EDM is expected to be minuscule for baryons and leptons in the SM and any observation would imply the existence of physics beyond the SM [10-12]. There are no direct measurements to date of such properties for charm and beauty baryons, and also for the $\tau$ lepton, due to the difficulties imposed by their short lifetimes. For the charm quark (chromo-)EDM only indirect limits exist based on the experimental bounds on the neutron EDM $[13,14]$.

Recently it has been proposed to measure charm baryon $\mathrm{MDM} / \mathrm{EDM}$ at a fixed-target experiment to be installed at the Large Hadron Collider (LHC) [15-19], following an experiment on the $\Sigma^{+}$hyperon MDM [20,21] and other proposals at Fermilab $[22,23]$. By exploiting the phenomenon of particle channeling in bent crystals, the electric and magnetic dipole moments of short-lived particles can be measured by studying the spin precession induced by the intense electric field between the crystal atomic planes, first proposed by Baryshevsky in 1979 [24]. In a crystal, the 
strong electric field experienced by a positively charged particle in the proximity of the atomic planes exerts a strong force and the particle trajectory becomes confined within two crystalline planes. This phenomenon, called planar channeling, can occur if the entrance angle between the particle trajectory and a crystal plane is lower than the Lindhard critical angle for channeling, $\theta_{L}=\sqrt{2 U_{0} /(p \beta c)}$, where $U_{0}$ is the potential-well depth, $p$ the particle momentum and $\beta$ its velocity $[25,26]$. For a $1 \mathrm{TeV}$ charged particle $\theta_{L} \approx 6.3 \mu \mathrm{rad}\left(\theta_{L} \approx 7.7 \mu \mathrm{rad}\right)$ in a silicon (germanium) crystal. The $\Lambda_{c}^{+}$and $\Xi_{c}^{+}$charm baryons, produced by interactions of the $7 \mathrm{TeV}$ LHC proton beam on a fixed target, are allowed to have initial polarization perpendicular to the production plane, due to parity symmetry conservation in strong interactions. The generation occurs within a cone with aperture $1 / \gamma \approx 1 \mathrm{mrad}$, where $\gamma$ is the Lorentz factor of the charm baryon. After the target, a bent crystal is placed to act as a special type of spectrometer deflecting channeled particles of a constant angle $\theta_{C}$ within the detector acceptance. The upgraded LHCb detector is particularly suited for this experiment thanks to its forward geometry and excellent performance for the reconstruction of heavy hadrons $[27,28]$. However, only a small fraction of the produced charm baryons, entering the crystal at an angle within the critical angle, is channeled and deflected. A sizable spin precession, induced by the intense electromagnetic field between crystal atomic planes, allows to probe for electromagnetic dipole moments, as illustrated in Fig. 1. For ultrarelativistic channeled particles with $\gamma \gg 1$, the spin precession angle induced by the MDM in the $y z$ plane is

$$
\Phi \approx \frac{g-2}{2} \gamma \theta_{C}
$$

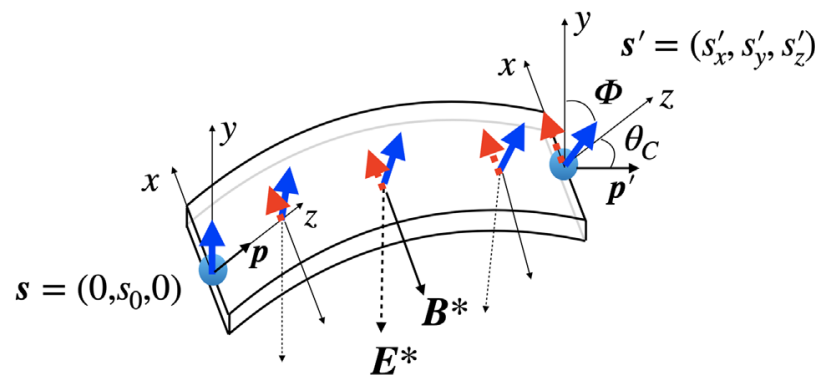

FIG. 1. Deflection of a particle with initial and final momentum $\boldsymbol{p}$ and $\boldsymbol{p}^{\prime}$, and spin-polarization precession in the $y z$ and $x z$ plane induced by the MDM and the EDM, respectively. The solid (blue) arrow represents the initial spin-polarization vector $s$, aligned along the $y$ axis in this example, normal to the crystal plane at entry. The spin is rotated of an angle $\Phi$ to $s^{\prime}$ after channeling in a crystal with bending angle $\theta_{C}$. The dashed (red) arrow indicates the (magnified) $s_{x}^{\prime}$ spin polarization component proportional to the particle EDM. The $\boldsymbol{B}^{*}\left(\boldsymbol{E}^{*}\right)$ indicates the magnetic (electric) field in the particle rest frame. where $\theta_{C}=L / \rho$ is the crystal bending angle, $L$ is the length of the crystal arc and $\rho$ the curvature radius. The presence of a nonzero EDM, in the limit of $d \ll g-2$, introduces a spin-polarization component $s_{x}^{\prime}$ perpendicular to the plane of the crystal bending [17],

$$
s_{x}^{\prime} \approx s_{0} \frac{d}{g-2}(1-\cos \Phi),
$$

where $s_{0}$ is the initial polarization. According to previous studies, a germanium crystal allows for enhanced sensitivity to MDM and EDM with respect to the silicon crystal $[18,29]$ due to the higher electric field between crystal atomic planes.

In this article, the design and characterization on beam of the first silicon and germaniun bent crystal prototypes for charm baryon spin precession are reported in Sec. II. Advanced experimental techniques and sensitivities studies for optimal future measurements of charm baryon dipole moments are discussed in Secs. III and IV, respectively. The main improvements with respect to previous studies [18] include a full amplitude analysis for optimal determination of baryon polarization, a more realistic polarization model [19], an optimized target thickness, CRYSTALRAD Monte Carlo simulations for particle channeling also for cryogenic temperatures [30], and additional charm baryon decay modes.

\section{BENT CRYSTAL PROTOTYPES AND TEST ON BEAM}

Crystal-assisted efficient steering of high-energy particle beams requires bent crystals with a tight control over their deformational state and an exceptionally low number of crystalline defects. Nonuniformities of the deformational state of the crystal and crystalline defects would indeed lead particles to be lost from channeling condition, lowering the efficiency of the steering process [26,31].

Crystals are manufactured through a revisitation of a protocol [32-34] already assessed for manufacturing of crystals of few mm length suitable for steering of particle beams circulating at the LHC [35]. The prime materials are a (111) oriented $5 \mathrm{~mm}$ thick silicon wafer and a (110) oriented $1 \mathrm{~mm}$ thick germanium wafer. With the aim to maintain an optimal steering efficiency, wafers with less than $1 / \mathrm{cm}^{2}$ dislocation density were selected from a large stock of wafers.

Mechanical dicing was applied to wafers to obtain a $50 \times 5 \times 80 \mathrm{~mm}^{3}$ crystal and a $35 \times 1 \times 55 \mathrm{~mm}^{3}$ crystal made of silicon and germanium, respectively. The first value indicates the dimension along the beam and in both cases was chosen according to Ref. [18]. Each crystal was mechanically bent along this longer dimension through a bender properly shaped to impart to the crystal the nominal bending radius of $5.0 \mathrm{~m}$ for silicon and $3.6 \mathrm{~m}$ for germanium, respectively. Given the nominal bending 


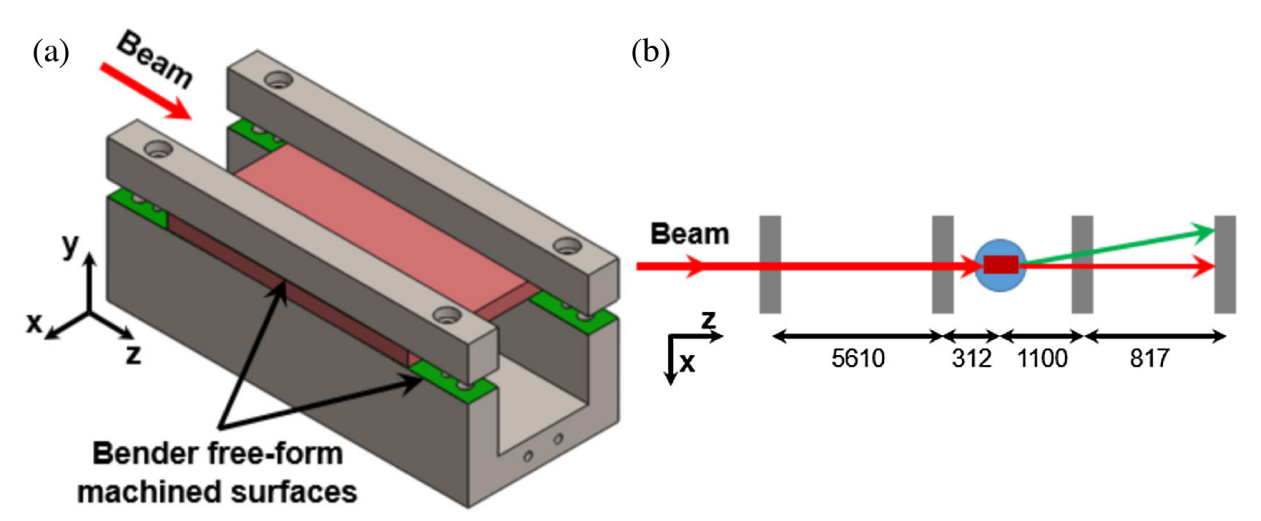

FIG. 2. (a) Sketch of an assembly made of crystal (red part) and associated bender (gray parts). Surfaces in contact with the crystal (green color) have been machined to a free-form surface. As the crystal is clamped in the bender, it bends around the $x$ axis. A particle beam, initially propagating along the $z$ axis, is channeled between bent atomic planes and deflected. (b) Sketch of the experimental setup at the CERN H8 beam line. A $180 \mathrm{GeV}$ positive hadron beam is directed to the crystal. The trajectory of each particle is reconstructed before and after interaction with the crystal by a tracking telescope (gray boxes). The crystal (red rectangle) is mounted on a high-resolution goniometer (blue circle). As the crystal is oriented in order to channel the incoming beam between its atomic planes, beam steering occurs and a fraction of the beam is deflected (green arrow). Distances between elements of the setup are expressed in $\mathrm{mm}$.

radius, channeled particles are deflected at an angle equal to $16 \mathrm{mrad}$ for silicon and $15 \mathrm{mrad}$ for germanium, see Fig. 2. The uniformity of the crystal deformation plays a key role for obtaining the expected steering efficiency: to enhance uniformity of crystal curvature, the nominal shape of the surfaces of the bender in contact with the crystal have been optimized through finite element models to free-form surfaces. After bending, the crystal deformational state was characterized [36] by means of high-resolution diffraction of a $8 \mathrm{keV}$ x-ray beam.

At the H8 external beam line of the Super Proton Synchrotron (SPS) at CERN, the crystal/bender assemblies were mounted on a goniometer capable of rotations with accuracy of $1 \mu \mathrm{rad}$ and aligned to a $180 \mathrm{GeV}$ positively charged hadron beam in order to measure particle channeling. The beam angular divergence (26 $\mu \mathrm{rad})$ resulted to be wider than $\theta_{L}$ for $180 \mathrm{GeV}$ particles $(\approx 15 \mu \mathrm{rad}$ for silicon and $\approx 17 \mu \mathrm{rad}$ for germanium), also the dimension along the $y$ coordinate $(\approx 8 \mathrm{~mm})$ was larger than the geometrical size of the crystal. To select particle trajectories intercepting the crystal and impinging on bent atomic planes at angle comparable to $\theta_{L}$, a tracking telescope based on four microstrips detectors was used. Two detectors were placed before and two were placed after the crystal to reconstruct particle trajectories before and after the interaction. Monte Carlo simulations of the beam test setup, based on the GEANT4 toolkit [37], are used to determine the angular uncertainty on particle trajectories before and after the interaction with the crystal, which resulted to be $\approx 7$ and $\approx 50 \mu \mathrm{rad}$, respectively.

The angular distribution of the beam after interaction with the crystal is shown in Fig. 3(a). The peak of the distribution on the left is populated by particles which are not channeled between atomic planes at the crystal entry face [38] and by particles which are initially channeled but with an impact parameter with respect to atomic planes smaller than the thermal vibration amplitude of the atoms of the crystal. For such particles, single scattering events with the inner shell electrons of the atoms or with the atomic nuclei result in a drastic change of their trajectory, leading them out of channeling regime soon after being channeled [39]. The peaks on the right, populated by channeled particles over the entire length of the crystal, are centered at angles of $15988 \pm 5$ and $14670 \pm 2 \mu \mathrm{rad}$ for the case of silicon and germanium crystals, respectively. Given the fact that in the final application the crystal would interact with a particle beam whose divergence is much larger than the critical angle for channeling, the steering efficiency of the crystal is measured for particles reaching the crystal at a nominal angle of $\pm 30 \mu \mathrm{rad}$, corresponding to an angular window about 2 times larger than the critical angle for channeling, and with deflection angle in a window of $\pm 500 \mu \mathrm{rad}$ around the peak of maximum deflection. Within that angular window, the efficiencies are measured according to Ref. [40]. Table I summarizes the most important features of the crystal prototypes and the measured channeling efficiencies. Using Monte Carlo simulations for the interaction between particle beam and crystal (for details see Appendix B 1), the steering efficiency as a function of the energy of the beam is determined for the two crystals assuming a uniform bending radius. Steering efficiency results are shown in Fig. 3(b).

\section{EXPERIMENTAL TECHNIQUES}

Charm baryon MDM and EDM measurements require advanced analysis techniques discussed below. 


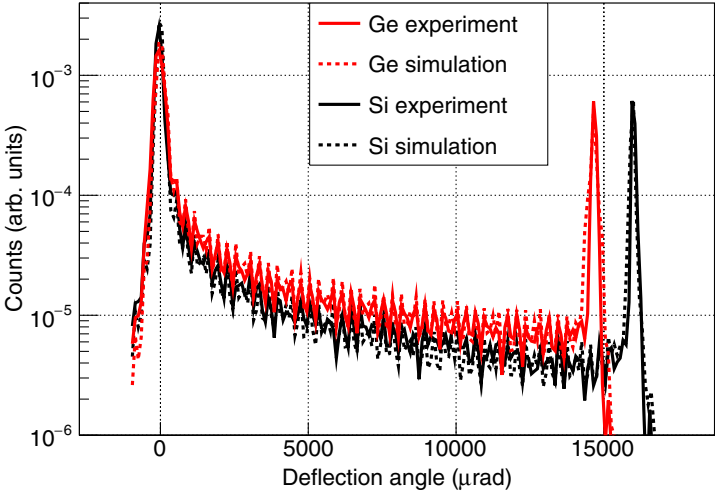

(a)

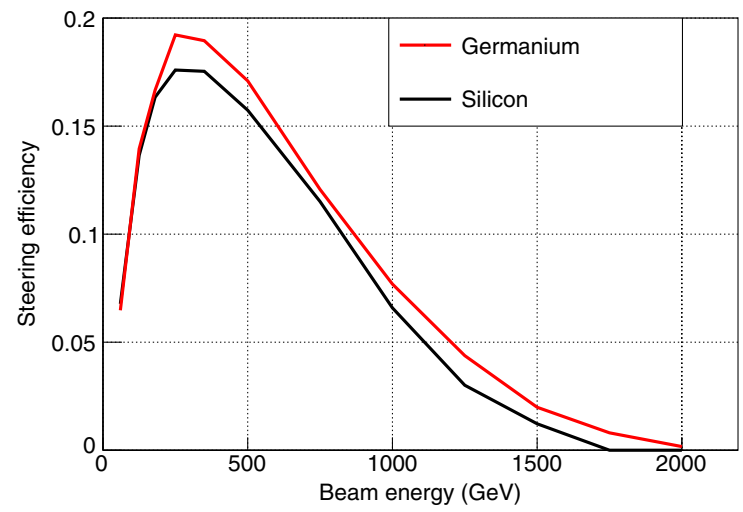

(b)

FIG. 3. (a) Angular distributions of a $180 \mathrm{GeV}$ hadron beam after interaction with the silicon (black lines) and germanium (red lines) crystals. Experimental data (solid curves) are compared to results of Monte Carlo simulations (dashed curves). The maxima located on the right side of the plot correspond to particles that are deflected, being channeled over the entire crystal length. The oscillations between the two peaks are due to the discrete structure of the strip detectors. By selecting particles impinging on the crystal within $\pm 30 \mu \mathrm{rad}$, steering efficiencies of $(8.9 \pm 0.5) \%$ and $(10.8 \pm 0.5) \%$ are measured for silicon and germanium crystals, respectively. (b) Monte Carlo simulation results for the steering efficiency of the two crystals as a function of the energy of the beam, assuming uniform bending radius and a particle beam with uniform angular distribution at the crystal entrance.

Combining these with an optimized experimental setup, the sensitivity to the observables of interest is extracted. To that end, large samples of pseudoexperiments are used, where the kinematics of $\Lambda_{c}^{+}$and $\Xi_{c}^{+}$baryons produced in the target are generated according to their momentum spectra as obtained from PYTHIA 8.244 event generator [41] tuned to NNPDF3.1sx NNLO NLLx LUXQED $\alpha_{s}\left(m_{Z}\right)=0.118$ parton distribution functions $42,43]$ including LHCb charm production data [44].

As a case study, the $\Lambda_{c}^{+} \rightarrow p K^{-} \pi^{+}$baryon decay is discussed. In the helicity formalism [45] the transition amplitude between an initial state with spin projection $m_{\Lambda_{c}^{+}}$ along the quantization axis and a final state with proton helicity $m_{p}$ is indicated as $\mathcal{A}_{m_{\Lambda_{c}^{+}}, m_{p}}(\boldsymbol{\xi})$, as a function of the phase space variables $\xi$. The decay distribution can be written as

$$
W(\boldsymbol{\xi} \mid s)=f(\boldsymbol{\xi})+s g(\boldsymbol{\xi}),
$$

where $s$ is the spin-polarization magnitude along a given axis, and the functions $f(\boldsymbol{\xi})$ and $g(\boldsymbol{\xi})$ are determined by the decay amplitudes $\mathcal{A}_{m_{\Lambda_{c}^{+}}, m_{p}}(\boldsymbol{\xi})$ as

$$
\begin{aligned}
f(\boldsymbol{\xi}) & =W(\boldsymbol{\xi} \mid s=0) \\
& \propto \sum_{m_{p}= \pm 1 / 2}\left[\left|\mathcal{A}_{1 / 2, m_{p}}(\boldsymbol{\xi})\right|^{2}+\left|\mathcal{A}_{-1 / 2, m_{p}}(\boldsymbol{\xi})\right|^{2}\right], \\
g(\boldsymbol{\xi}) & =\frac{1}{2}[W(\boldsymbol{\xi} \mid s=1)-W(\boldsymbol{\xi}, s=-1)] \\
& \propto \sum_{m_{p}= \pm 1 / 2}\left[\left|\mathcal{A}_{1 / 2, m_{p}}(\boldsymbol{\xi})\right|^{2}-\left|\mathcal{A}_{-1 / 2, m_{p}}(\boldsymbol{\xi})\right|^{2}\right],
\end{aligned}
$$

and satisfy the normalization conditions

$$
\int f(\boldsymbol{\xi}) d \boldsymbol{\xi}=1, \quad \int g(\boldsymbol{\xi}) d \boldsymbol{\xi}=0 .
$$

The value of the average event information $S^{2}$ represents the sensitivity to the polarization $s$ that can be computed as [46]

$$
S^{2}=\int \frac{g^{2}(\boldsymbol{\xi})}{f(\boldsymbol{\xi})+s_{0} g(\boldsymbol{\xi})} d \boldsymbol{\xi}
$$

where $s_{0}$ is the best estimate for the polarization whose variance is $\sigma_{s}^{2}=\left(N S^{2}\right)^{-1}$, with $N$ the number of signal

TABLE I. Geometrical parameters of silicon and germanium crystals used in the experiment, and recorded channeling efficiencies compared to expected from Monte Carlo simulations. The length (thickness) represents the longitudinal (transverse) dimension of the crystal with respect to the beam direction. The uncertainties on the steering efficiency results take into account both statistical and systematic effects.

\begin{tabular}{lccccrrr}
\hline \hline & & & \multicolumn{2}{c}{ Deflection angle $(\mathrm{mrad})$} & & \multicolumn{2}{c}{ Steering efficiency $(\%)$} \\
& Length $(\mathrm{mm})$ & Thickness $(\mathrm{mm})$ & X-ray & Channeling & & Measured & Simulation \\
\hline Germanium & 55 & 1 & $14.5 \pm 0.8$ & $14.670 \pm 0.002$ & & $10.8 \pm 0.5$ & $12.3 \pm 0.5$ \\
Silicon & 80 & 5 & $16.1 \pm 0.8$ & $15.988 \pm 0.005$ & & $8.9 \pm 0.5$ & $9.9 \pm 0.5$ \\
\hline \hline
\end{tabular}




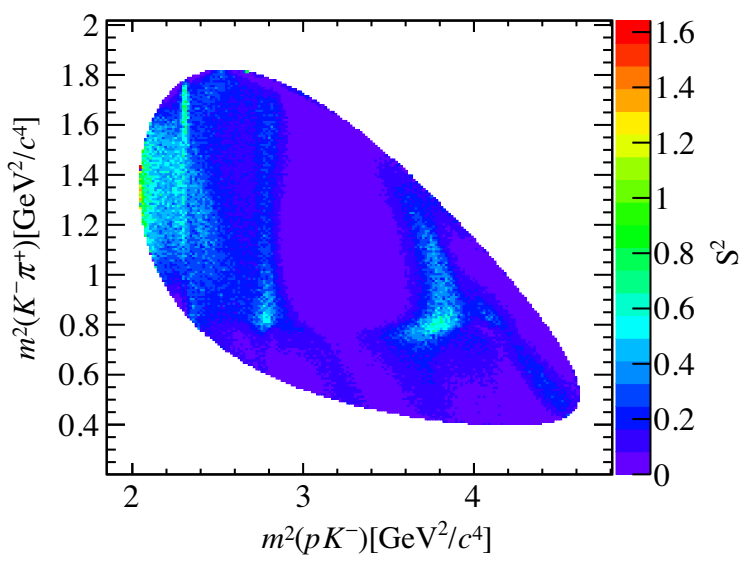

FIG. 4. Average event information $S^{2}$ to the $\Lambda_{c}^{+}$baryon polarization as a function of the Dalitz plot position defined by the squared invariant masses $m^{2}\left(p K^{-}\right)$and $m^{2}\left(K^{-} \pi^{+}\right)$, as obtained from a large statistics pseudoexperiment.

events. The $\Lambda_{c}^{+} \rightarrow p K^{-} \pi^{+}$decay amplitude is dominated by the sum of quasi-two-body $p K^{*}, \Lambda^{*} \pi^{+}$, and $\Delta^{++} K^{-}$ resonant contributions $[47,49]$. The average event information for $s_{0}=0$, based on a pseudoexperiment, is shown in Fig. 4 as a function of the Dalitz plot position, defined by the squared invariant masses $m^{2}\left(p K^{-}\right)$and $m^{2}\left(K^{-} \pi^{+}\right)$, with an average value $S^{2} \approx 0.145$ [50]. This result can be compared with a two-body decay, where $S^{2}$ for $s_{0}=0$ is related to the effective decay asymmetry parameter $\alpha_{\text {eff }}$, which characterizes parity violation, $S^{2}=\alpha_{\text {eff }}^{2} / 3$, and it is found to be compatible to that estimated for the $\Lambda_{c}^{+} \rightarrow$ $\Delta^{++} K^{-}$decay using the known value $\alpha_{\Delta^{++} K^{-}}=-0.67 \pm$ 0.30 [17], affected by large uncertainty. Hence, the full amplitude analysis of the $\Lambda_{c}^{+} \rightarrow p K^{-} \pi^{+}$decay enables the measurement of the $\Lambda_{c}^{+}$spin precession using three-body decay modes [50,51], which effectively increases the statistics for the dipole moments measurement by a factor $\mathcal{B}\left(\Lambda_{c}^{+} \rightarrow p K^{-} \pi^{+}\right) / \mathcal{B}\left(\Lambda_{c}^{+} \rightarrow \Delta^{++} K^{-}\right) \approx 6$ with respect to that assumed in previous studies [17-19,29].

Additional $\Lambda_{c}^{+}$three- and four-body decays can be exploited for the measurement of the dipole moments in order to further increase the signal yield up to an additional factor of 3 , assuming similar average event information $S^{2}$ for all decays. This can be done analogously for $\Xi_{c}^{+}$baryon decays. In Tables II and III are listed the decay modes considered for the sensitivity studies with corresponding effective branching fractions, taking into account the ability to reconstruct the final state charged particles with the $\mathrm{LHCb}$ detector. Due to the high momentum of charm baryons produced in the fixed-target interactions, a large fraction of the long-lived $\Sigma^{+}, \Sigma^{-}$and $\Xi^{-}$strange baryons traverse the entire LHCb tracking system before decaying, thus their trajectories can be reconstructed as for stable charged particles. The crystal bending angle and length determine the momentum spectrum of channeled charm baryons and consequently
TABLE II. List of $\Lambda_{c}^{+}$baryon decay modes and corresponding absolute branching fraction $\mathcal{B}$ [47], efficiency for $\mathrm{LHCb}$ reconstructibility of the three-charged particles $\epsilon_{3 \text { trk }}$, and the effective branching fraction $\mathcal{B}_{\text {eff }}=\mathcal{B} \times \epsilon_{3 \text { trk }}$. The efficiency and effective branching fraction depend on the crystal parameters, here for germanium at room temperature with deflection angle of $16 \mathrm{mrad}$ and $10 \mathrm{~cm}$ length.

\begin{tabular}{lccc}
\hline \hline$\Lambda_{c}^{+}$final state & $\mathcal{B}(\%)$ & $\epsilon_{3 \text { trk }}$ & $\mathcal{B}_{\text {eff }}(\%)$ \\
\hline$p K^{-} \pi^{+}$ & $6.28 \pm 0.32$ & 0.99 & 6.25 \\
$\Sigma^{+} \pi^{-} \pi^{+}$ & $4.50 \pm 0.25$ & 0.54 & 2.43 \\
$\Sigma^{-} \pi^{+} \pi^{+}$ & $1.87 \pm 0.18$ & 0.71 & 1.33 \\
$p \pi^{-} \pi^{+}$ & $0.461 \pm 0.028$ & 1.00 & 0.46 \\
$\Xi^{-} K^{+} \pi^{+}$ & $0.62 \pm 0.06$ & 0.73 & 0.45 \\
$\Sigma^{+} K^{-} K^{+}$ & $0.35 \pm 0.04$ & 0.51 & 0.18 \\
$p K^{-} K^{+}$ & $0.106 \pm 0.006$ & 0.98 & 0.11 \\
$\Sigma^{+} \pi^{-} K^{+}$ & $0.21 \pm 0.06$ & 0.54 & 0.11 \\
$p K^{-} \pi^{+} \pi^{0}$ & $4.46 \pm 0.30$ & 0.99 & 4.43 \\
$\Sigma^{+} \pi^{-} \pi^{+} \pi^{0}$ & 3.20 & 0.54 & 1.72 \\
$\Sigma^{-} \pi^{+} \pi^{+} \pi^{0}$ & $2.1 \pm 0.4$ & 0.71 & 1.49 \\
$\Sigma^{+}\left[p \pi^{0}\right] \pi^{-} \pi^{+}$ & 2.32 & 0.46 & 1.06 \\
$\Sigma^{+}\left[p \pi^{0}\right] K^{-} K^{+}$ & 0.18 & 0.46 & 0.08 \\
$\Sigma^{+}\left[p \pi^{0}\right] \pi^{-} K^{+}$ & 0.11 & 0.46 & 0.05 \\
All & $\ldots$ & $\ldots$ & 20.2 \\
\hline \hline
\end{tabular}

the fraction of strange baryons reconstructed as stable charged particles. This requires that final state particles travel more than $9.4 \mathrm{~m}$ from their production point positioned $1.2 \mathrm{~m}$ upstream of the nominal $p p$ interaction region. Despite the lower production rate of the $\Xi_{c}^{+}$ compared to the $\Lambda_{c}^{+}$baryon, estimated to be about

TABLE III. List of $\Xi_{c}^{+}$baryon decay modes and corresponding relative branching absolute $\Xi_{c}^{+} \rightarrow \Xi^{-} \pi^{+} \pi^{-}$branching fraction $\mathcal{B}$ [52], estimate of the efficiency for $\mathrm{LHCb}$ reconstructibility of the three-charged particles $\epsilon_{3 \text { trk }}$, and the effective branching fraction $\mathcal{B}_{\text {eff }}=\mathcal{R} B \times \mathcal{B}\left(\Xi_{c}^{+} \rightarrow \Xi^{-} \pi^{+} \pi^{-}\right) \times \epsilon_{3 \text { trk }}$ or $\mathcal{B}_{\text {eff }}=\mathcal{B} \times \epsilon_{3 \text { trk }}$ where $\mathcal{B}$ measurement is available. The reported $\epsilon_{3 \text { trk }}$ and $\mathcal{B}_{\text {eff }}$ are for a $16 \mathrm{mrad}$ bent, $10 \mathrm{~cm}$ long germanium crystal at room temperature.

\begin{tabular}{lcccc}
\hline \hline$\Xi_{c}^{+}$final state & $\mathcal{R} B$ & $\mathcal{B}(\%)$ & $\epsilon_{3 \text { trk }}$ & $\mathcal{B}_{\text {eff }}(\%)$ \\
\hline$\Xi^{-} \pi^{+} \pi^{+}$ & 1 & $2.86 \pm 1.27$ & 0.64 & 1.84 \\
$\Sigma^{+} K^{-} \pi^{+}$ & $0.94 \pm 0.10$ & $\ldots$ & 0.42 & 1.14 \\
$\Sigma^{+} \pi^{-} \pi^{+}$ & $0.48 \pm 0.20$ & $\ldots$ & 0.44 & 0.60 \\
$p K^{-} \pi^{+}$ & $0.21 \pm 0.04$ & $\ldots$ & 0.99 & 0.60 \\
$\Sigma^{-} \pi^{+} \pi^{+}$ & $0.18 \pm 0.09$ & $\ldots$ & 0.61 & 0.31 \\
$\Sigma^{+} K^{-} K^{+}$ & $0.15 \pm 0.06$ & $\ldots$ & 0.41 & 0.18 \\
$\Omega^{-} K^{+} \pi^{+}$ & $0.07 \pm 0.04$ & $\ldots$ & 0.42 & 0.08 \\
$\Sigma^{+}\left[p \pi^{0}\right] K^{-} \pi^{+}$ & 0.48 & $\ldots$ & 0.57 & 0.79 \\
$\Sigma^{+}\left[p \pi^{0}\right] \pi^{-} \pi^{+}$ & 0.25 & $\ldots$ & 0.57 & 0.40 \\
$\Sigma^{+}\left[p \pi^{0}\right] K^{-} K^{+}$ & 0.08 & $\ldots$ & 0.59 & 0.13 \\
All & $\ldots$ & $\ldots$ & $\ldots$ & 6.1 \\
\hline \hline
\end{tabular}


$70 \%$ [18], and its smaller effective branching ratio, similar signal yields are expected for both baryon types decaying after the bent crystal due to their different lifetimes, about a factor of 2 longer for the $\Xi_{c}^{+}$baryon.

When there is a $\pi^{0}$ meson in the final state, a partial reconstruction of the decay can be performed based on charged particles only (for details see Appendix B 3). The charm baryon invariant mass is reconstructed using the corrected mass [53], the charm hadron flight length and direction are determined by reconstructing the production and the decay vertex positions, and its momentum can be estimated without bias by reconstructing the decay kinematics. Similarly, the average charm baryon spinpolarization vector can also be measured with no bias employing the reconstructed kinematics, and ultimately using a technique based on templates for full positive and negative polarization, similar to that for the measurement of the polarization of the $\tau$ leptons where the neutrinos are not reconstructed $[46,54]$.

The charm baryon polarization is perpendicular to the production plane, defined by the momenta of the impinging proton and that of the outgoing $\Lambda_{c}^{+}$baryon, and is parametrized as a function of its transverse momentum $p_{\mathrm{T}}$ with respect to the direction of the impinging proton as

$$
s_{0}\left(p_{\mathrm{T}}\right) \approx A\left(1-e^{-B p_{T}^{2}}\right),
$$

with $A \approx-0.9$ and $B \approx 0.4(\mathrm{GeV} / c)^{-2}$. The parametrization is based on a phenomenological dependence [48] used to describe the experimental results [49]. The $\Lambda_{c}^{+}$and $\Xi_{c}^{+}$ baryon polarization versus $p_{\mathrm{T}}$ can be measured precisely in fixed-target collisions at $\mathrm{LHCb}$ using the SMOG system $[55,56]$ to further improve the polarization model.

\section{SENSITIVITY STUDIES AND SETUP OPTIMIZATION}

The sensitivity to dipole moments is studied using pseudoexperiments and PYTHIA simulations for different angles between the impinging proton direction and the crystal orientation. The crystal reference frame $(x, y, z)$ is rotated at different angles $\theta_{y, C}$ around the $x_{L}$ axis in the $\left(x_{L}, y_{L}, z_{L}\right)$ laboratory frame [29], with $x$ parallel to the $x_{L}$ axis and $z_{L}$ parallel to the impinging proton direction. For nonzero $\theta_{y, C}$ values an initial polarization along the $x$ and $y$ axes is induced,

$$
\boldsymbol{s}=\left(s_{x}, s_{y}, 0\right) \approx \frac{s_{0}\left(p_{\mathrm{T}}\right)}{p_{\mathrm{T}}}\left(-p_{y_{L}}, p_{x_{L}}, 0\right),
$$

where $p_{x_{L}}$ and $p_{y_{L}}=p \sin \theta_{y, C}$ are the transverse momentum components along the laboratory $x_{L}$ and $y_{L}$ axis, respectively, and $p$ is the total momentum of the charm baryon. This is a consequence of parity conservation in strong interactions that forces the $\Lambda_{c}^{+}$polarization vector to be perpendicular to the production plane, defined by the proton and the $\Lambda_{c}^{+}$baryon momenta. A probability density function based upon the quasi-two-body approximation for the decay rate,

$$
\mathcal{W} \propto 1+\alpha_{\mathrm{eff}} \boldsymbol{s}^{\prime} \cdot \hat{\boldsymbol{k}}
$$

is used in order to effectively reproduce the fit procedure for data, where $\hat{\boldsymbol{k}}$ is the direction of the resonant intermediate state in the charm baryon helicity frame

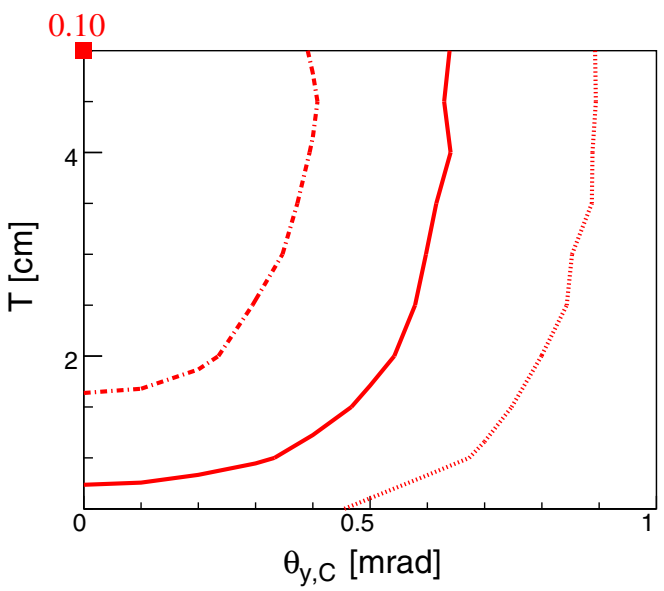

(a)

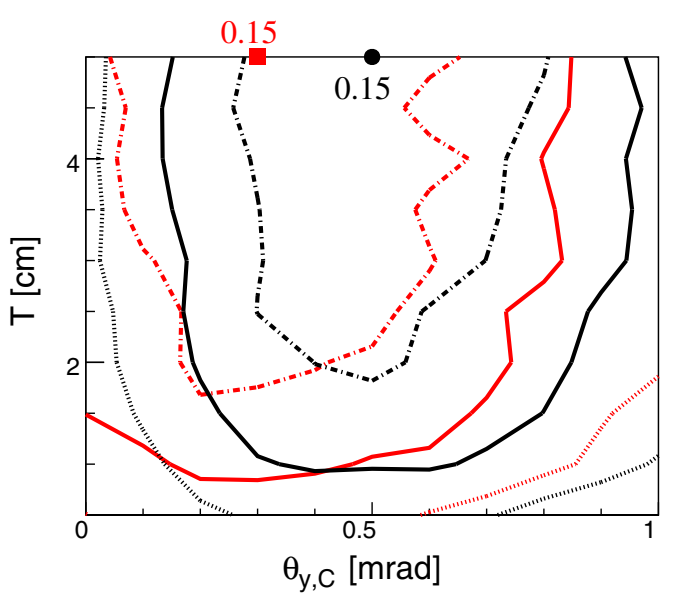

(b)

FIG. 5. Regions of minimal uncertainty of the $g$ (red curves) and $d$ (black) factors as a function of the crystal orientation angle $\theta_{y, C}$ and the target thickness $T$, for (a) MDM measurement alone and (b) combined MDM and EDM measurement, with magnetic anomalous moment $a \approx-0.03$ and $\Lambda_{c}^{+} \rightarrow p K^{-} \pi^{+}$decays. The markers and values represent the minimum uncertainty on the $g$ and $d$ factors relative to $1.37 \times 10^{13} \mathrm{PoT}$, corresponding to two years of data taking at a rate of $10^{6} \mathrm{p} / \mathrm{s}$ [60], using a $16 \mathrm{mrad}$ bent, $10 \mathrm{~cm}$ long germanium crystal at room temperature. The curves are the regions whose uncertainties are increased by $20 \%$, $50 \%$ and $100 \%$ with respect to the minimum. A similar behavior is observed for other considered crystal configurations. 
TABLE IV. Relevant parameters for $\Lambda_{c}^{+}$and $\Xi_{c}^{+}$baryons considered for sensitivity studies: the average event information $S^{2}$ (assumed to be similar for all modes), the detector efficiency $\epsilon_{\mathrm{DET}}$, which includes trigger, reconstruction and selection criteria of signal events based upon the $3 h$ system, the baryon production cross section $\sigma$, the anomalous magnetic moment $a$ (two values are used from Ref. [8], the second is indicated in squared brackets), the gyroelectric factor $d=0$, the effective branching ratio $\mathcal{B}_{\text {eff }}$, the channeling efficiency $\epsilon_{\mathrm{CH}}$, which includes the efficiency of the particle to be trapped into channeling regime, the decay flight efficiency $\epsilon_{\mathrm{DF}}$ of the baryon within the crystal length, the number of reconstructed charm baryons $N_{\text {rec }}$, the average boost $\langle\gamma\rangle$ and transverse momentum $\left\langle p_{\mathrm{T}}\right\rangle$, the initial spin-polarization $s_{x}$, and the initial spin-polarization $s_{y}$ for events with positive $p_{x_{L}}\left(-s_{y}\right.$ for negative $\left.p_{x_{L}}\right)$. Uncertainties on the MDM and EDM, $\sigma_{\mu}$ and $\sigma_{\delta}$, respectively, are relative to $1.37 \times 10^{13}$ PoT. At LHCb, silicon and germanium bent crystals at room temperature, and germanium at $77 \mathrm{~K}$, are considered. For comparison, the $\mathrm{S}_{2}$ scenario is evaluated with germanium at room temperature. The corresponding crystal parameters are reported in the table. The target thickness is $2 \mathrm{~cm}$ in all cases. All three- and four-body modes from Tables II and III are considered.

\begin{tabular}{|c|c|c|c|c|}
\hline$S^{2}$ & \multicolumn{4}{|c|}{$\approx 0.145$} \\
\hline$\epsilon_{\mathrm{DET}}(\%)$ & \multicolumn{4}{|c|}{0.20} \\
\hline Crystal configuration & $\mathrm{Ge}$ & $\mathrm{Si}$ & Ge $77 \mathrm{~K}$ & $\mathrm{Ge}_{2}$ \\
\hline angle (mrad) & 16 & 16 & 16 & 7 \\
\hline length $(\mathrm{cm})$ & 10 & 10 & 10 & 7 \\
\hline \multicolumn{5}{|c|}{$\Lambda_{c}^{+}$baryon } \\
\hline$\sigma(\mu \mathrm{b} /$ nucleon $)$ & \multirow{2}{*}{\multicolumn{4}{|c|}{$\begin{array}{c}10.6 \\
\approx-0.03[-0.76]\end{array}$}} \\
\hline$a=(g-2) / 2$ & & & & \\
\hline$d$ & \multicolumn{4}{|c|}{0} \\
\hline $\mathcal{B}_{\text {eff }}(\%)$ & 20.2 & 19.5 & 20.6 & 20.6 \\
\hline$\epsilon_{\mathrm{CH}}\left(\times 10^{-4}\right)$ & 4.5 & 2.7 & 8.7 & 11.1 \\
\hline$\epsilon_{\mathrm{DF}}\left(\times 10^{-2}\right)$ & 3.3 & 1.7 & 4.9 & 13.1 \\
\hline$N_{\text {rec }}$ & 586 & 181 & 1748 & 5879 \\
\hline$\langle\gamma\rangle$ & 709 & 573 & 834 & 855 \\
\hline$\left\langle p_{\mathrm{T}}\right\rangle\left(\mathrm{GeV} / c^{2}\right)$ & 0.79 & 0.71 & 0.86 & 0.87 \\
\hline$s_{x}(\%)$ & 11.8 & 8.6 & 14.1 & 15.6 \\
\hline$s_{y}(\%)$ & -15.3 & -14.2 & -16.1 & -16.1 \\
\hline$\sigma_{\mu}\left(\times 10^{-2} \mu_{\mathrm{N}}\right)$ & 1.6 & 3.4 & 0.8 & 0.9 \\
\hline$\sigma_{\delta}\left(\times 10^{-16} e \mathrm{~cm}\right)$ & $2.2[9.8]$ & $5.6[17.1]$ & $0.9[5.7]$ & $1.0[2.9]$ \\
\hline \multicolumn{5}{|c|}{$\Xi_{c}^{+}$baryon } \\
\hline$\sigma(\mu \mathrm{b} /$ nucleon $)$ & \multirow{3}{*}{\multicolumn{4}{|c|}{$\begin{array}{c}7.5 \\
\approx 0.05[-0.47] \\
0\end{array}$}} \\
\hline$a=(g-2) / 2$ & & & & \\
\hline$d$ & & & & \\
\hline $\mathcal{B}_{\text {eff }}(\%)$ & 6.1 & 5.8 & 6.2 & 6.2 \\
\hline$\epsilon_{\mathrm{CH}}\left(\times 10^{-4}\right)$ & 5.7 & 3.8 & 10.4 & 12.2 \\
\hline$\epsilon_{\mathrm{DF}}\left(\times 10^{-2}\right)$ & 10.7 & 7.6 & 13.0 & 24.6 \\
\hline$N_{\text {rec }}$ & 627 & 284 & 1429 & 3146 \\
\hline$\langle\gamma\rangle$ & 514 & 433 & 576 & 588 \\
\hline$\left\langle p_{\mathrm{T}}\right\rangle\left(\mathrm{GeV} / c^{2}\right)$ & 0.74 & 0.69 & 0.78 & 0.79 \\
\hline$s_{x}(\%)$ & 8.6 & 6.7 & 10.2 & 10.5 \\
\hline$s_{y}(\%)$ & -15.7 & -14.8 & -16.3 & -16.5 \\
\hline$\sigma_{\mu}\left(\times 10^{-2} \mu_{\mathbf{N}}\right)$ & 1.8 & 3.1 & 1.0 & 1.5 \\
\hline$\sigma_{\delta}\left(\times 10^{-16} e \mathrm{~cm}\right)$ & $3.0[5.1]$ & $5.9[6.8]$ & $1.5[3.5]$ & $2.4[2.1]$ \\
\hline
\end{tabular}

and $\boldsymbol{s}^{\prime}$ the spin-polarization vector after spin precession in the crystal,

$$
\begin{aligned}
& s_{x}^{\prime} \approx s_{y} \frac{a^{\prime} d^{\prime}}{a_{d}^{\prime 2}}(1-\cos \Phi)+s_{x}\left(\frac{a^{\prime 2}}{a_{d}^{\prime 2}}+\frac{d^{\prime 2}}{a_{d}^{\prime 2}} \cos \Phi\right), \\
& s_{y}^{\prime} \approx s_{y}\left(\frac{d^{\prime 2}}{a_{d}^{\prime 2}}+\frac{a^{\prime 2}}{a_{d}^{\prime 2}} \cos \Phi\right)+s_{x} \frac{a^{\prime} d^{\prime}}{a_{d}^{\prime 2}}(1-\cos \Phi), \\
& s_{z}^{\prime} \approx-s_{y} \frac{a^{\prime}}{a_{d}^{\prime}} \sin \Phi+s_{x} \frac{d^{\prime}}{a_{d}^{\prime}} \sin \Phi,
\end{aligned}
$$

where $a^{\prime}=a+1 /(1+\gamma)$, with $a=(g-2) / 2$ the anomalous magnetic moment, $d^{\prime}=d / 2, a_{d}^{\prime}=\sqrt{a^{\prime 2}+d^{\prime 2}}$, and $\Phi=\gamma \theta_{C} a_{d}^{\prime}$. These expressions hold at $\mathcal{O}\left(10^{-2}\right)$ precision and follow by solving the T-BMT equation [57-59] for initial polarization along the $x$ and $y$ crystal frame directions, according to the procedure described elsewhere $[17,18]$. The expected precession angle $\Phi$ and the initial polarization-vector $s$ can be estimated on event-by-event basis using the measured boost factor $\gamma$ and the transverse momentum of the charm baryon. 
Following this approach, an enhanced sensitivity is obtained while retaining the complete dependence on $g$, $d$ and $\gamma$, reducing significantly the need of a large crystal orientation angle $\theta_{y, C}$ for the EDM case [29], and solving discrete ambiguities in the simultaneous extraction of the dipole moments [18]. Pseudoexperiments have been generated assuming a proton flux on target of about $10^{6} \mathrm{p} / \mathrm{sec}$, which corresponds to $4.3 \times 10^{10}$ protons on target $(\mathrm{PoT})$ in ten hours operations of the LHC [60], about $1.37 \times 10^{13}$ PoT can be delivered by the accelerator in two years with an operational efficiency of about 55\%. In Fig. 5(a) the optimal configuration for the measurement of the MDM alone, i.e. using Eq. (10) with $d=0$, is obtained at $\theta_{y, C}=0$, which corresponds to the maximum initial polarization along the $y$ axis, perpendicular to the magnetic field $\boldsymbol{B}^{*}$. In Fig. 5(b), the optimal configuration for the combined measurement of MDM and EDM, i.e. using Eq. (10) with $d$ as free parameter, is obtained at $\theta_{y, C}$ different from zero. For the MDM the difference with respect to Fig. 5(a) is due to limited PoT sample and to correlations between the $a$ and $d$ parameters. The sensitivity to EDM requires a polarization component perpendicular to the electric field $\boldsymbol{E}^{*}$, e.g. initial polarization along the $x$ axis. However, as illustrated in Fig. 5(b), for small $a \approx-0.03$ [8], the decrease of EDM sensitivity with $\theta_{y, C} \approx 0.3(0) \mathrm{mrad}$ with respect to the maximal sensitivity at $0.5 \mathrm{mrad}$ amounts to about $20 \%$ (100\%), with marginal impact on the MDM (for further details see Appendix B 4).

A smaller crystal orientation angle reduces potential systematic uncertainties due to initial polarization along the crystal $x$ axis, mimicking EDM effects. Nevertheless, simulation studies show that a precise determination of the polarization model allows such systematic uncertainty to be kept under control. The study of charm baryons with opposite polarization and the use of up- and down-bending crystals, inducing opposite spin precession, offer complementary tools to prove the robustness of the results and control systematic uncertainties [61]. For large $a \approx-0.76$ [8] the choice of crystal orientation has no impact on the sensitivities.

In addition, in Fig. 5 the $\mathrm{W}$ target thickness is optimized considering the inelastic interactions of charm baryons within the target, regulated by the nuclear interaction length and the survival probability due to its lifetime [47] (for details see Appendix B 2). A W target thickness of $2 \mathrm{~cm}$, about a factor of two thinner than that where maximal $\Lambda_{c}^{+}$ and $\Xi_{c}^{+}$yields at the target exit are reached, is considered as operational value. With this choice the charm baryon dipole moment sensitivities are reduced by about $20 \%$ with respect to the optimal thickness reached around $5 \mathrm{~cm}$, as illustrated in Fig. 5 for $\Lambda_{c}^{+}$baryons, while detector occupancies are largely mitigated, as required for detector operation and safety.

Relevant parameters for the sensitivity studies are reported in Table IV, along with the expected signal yields

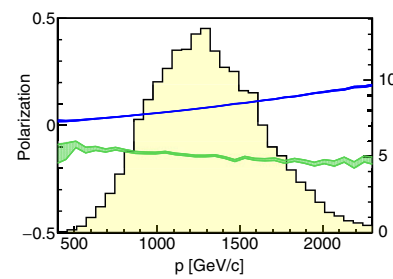

(a)

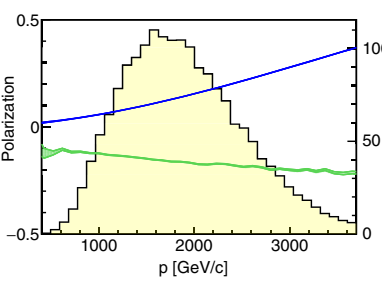

(c)

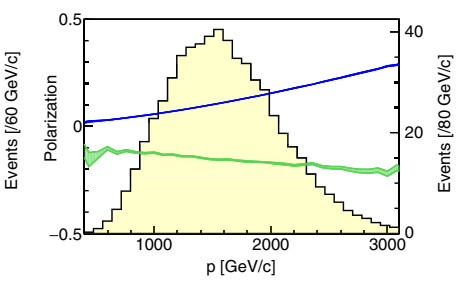

(b)

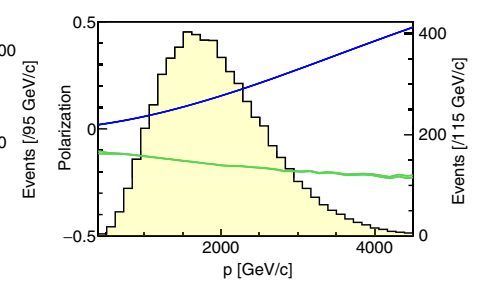

(d)
FIG. 6. Initial polarizations $s_{x}$ (hatched blue bands) and $s_{y}$ for events with positive $p_{x_{L}}\left(-s_{y}\right.$ for negative $p_{x_{L}}$, solid green) as a function of the $\Lambda_{c}^{+}$baryon momentum for (a) silicon $293 \mathrm{~K}$, (b) germanium $293 \mathrm{~K}$, (c) germanium $77 \mathrm{~K}$, and (d) germanium $293 \mathrm{~K}$ for $\mathrm{S}_{2}$, with parameters reported in the text. The bands represent one standard deviation regions from the pseudoexperiments. The histograms, normalized to $1.37 \times 10^{13}$ PoT, show the spectra of channeled and reconstructed particles.

and uncertainties on the MDM and EDM in two years of data taking with the LHCb detector, for different crystal configurations. Figures 6 and 7 show the corresponding reconstructed $\Lambda_{c}^{+}$and $\Xi_{c}^{+}$momentum spectra, along with the estimated $s_{x}$ and $s_{y}$ initial polarizations as a function of the baryon momentum. The latter are determined by the convolution of Eq. (8) with the transverse momentum distribution of channeled baryons. How the sensitivities evolve as a function of PoT is illustrated in Fig. 8.

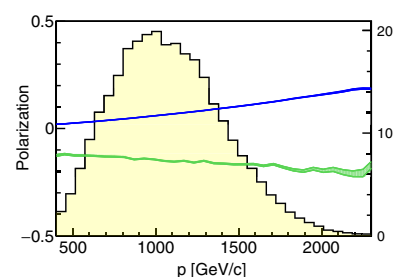

(a)

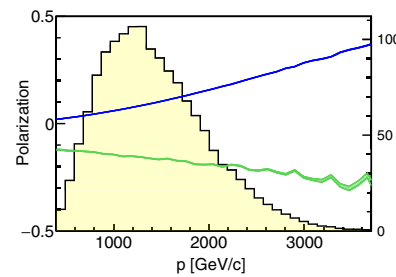

(c)

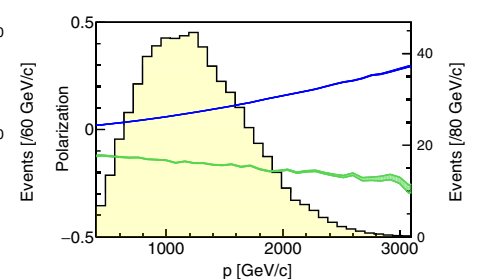

(b)

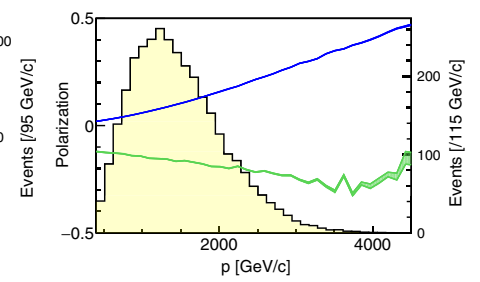

(d)
FIG. 7. Same as Fig. 6 for $\Xi_{c}^{+}$baryons. The oscillations in the initial polarization bands (particularly visible for $s_{y}$ ) are due to the limited sample size of the simulations. 


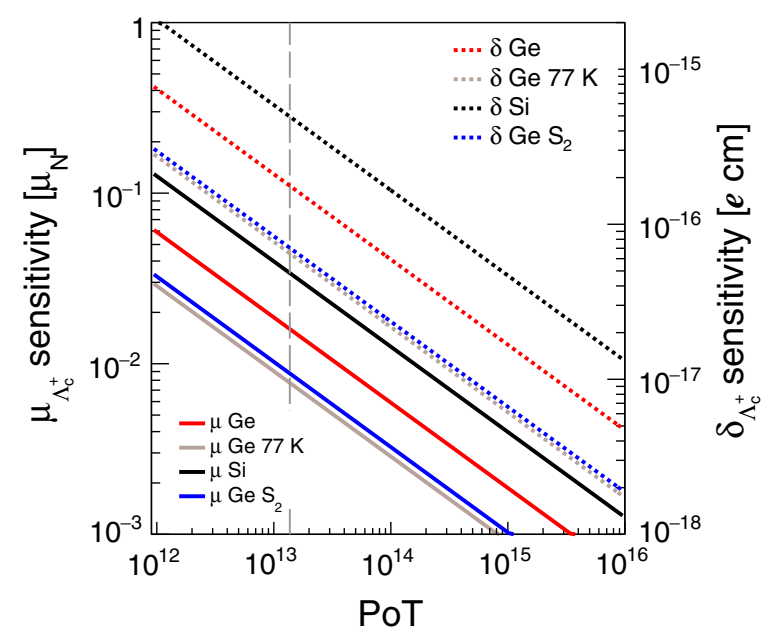

(a)

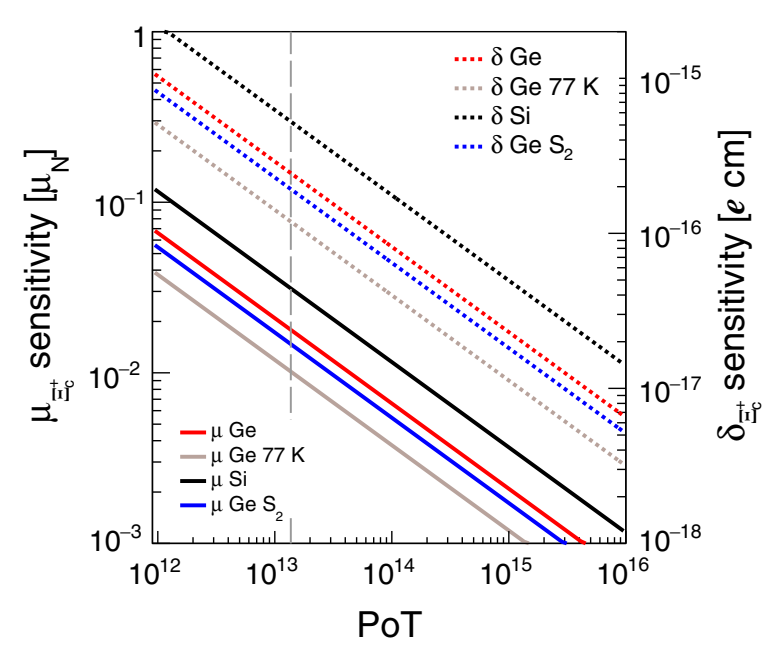

(b)

FIG. 8. Uncertainties on the MDM and EDM of $\Lambda_{c}^{+}$(a) and $\Xi_{c}^{+}$ (b) baryons as a function of PoT at $\mathrm{LHCb}$ and at a dedicated experiment $\left(\mathrm{S}_{2}\right)$ with increased forward acceptance. The sensitivities at $\mathrm{LHCb}$ for room temperature $293 \mathrm{~K}$ silicon and germanium bent crystals are compared with a germanium crystal cooled at $77 \mathrm{~K}$, with parameters reported in the text. All three- and four-body $\Lambda_{c}^{+}$and $\Xi_{c}^{+}$decays from Tables II and III are considered, with anomalous magnetic moment $a$ assumed to be $\approx-0.03$ and $\approx 0.05$, respectively. The vertical long-dashed lines refer to $1.37 \times 10^{13}$ PoT, corresponding to two years of data taking.

Silicon and germanium crystals with deflection angle of $16 \mathrm{mrad}$ and $10 \mathrm{~cm}$ length are considered for compatibility with the $\mathrm{LHCb}$ detector acceptance and operations, while maximizing experimental sensitivity. For the case of germanium, sensitivity results at $77 \mathrm{~K}$ in addition to room temperature are shown. The scenario of a future dedicated experiment at the LHC, indicated as $\mathrm{S}_{2}$, is also studied using a setup based on a germanium crystal at room temperature of $7 \mathrm{mrad}$ bending angle and $7 \mathrm{~cm}$ length, which would allow effective separation of channeled charm baryons from forwardly produced background particles, to be kept outside the detector acceptance. Identical detector performance and signal reconstruction efficiency to the $\mathrm{LHCb}$ apparatus are assumed, but extended in the forward direction. Nevertheless, in the $\mathrm{S}_{2}$ scenario channeled baryons are deflected at smaller angles with high momentum, extending up to about $4 \mathrm{TeV}$, and would require an advanced detector design with a long lever arm and intense magnetic field for precise particle momentum measurements, and high granularity to be able to operate and reconstruct the events under high background levels in the very forward region.

Compared to the configuration of germanium at room temperature, the significantly higher yields with germanium $S_{2}$, close to a factor of $10(5)$ for $\Lambda_{c}^{+}\left(\Xi_{c}^{+}\right)$baryons, and the $20 \%(15 \%)$ harder momentum spectrum, reflect in an increase of MDM sensitivity equivalent to a data sample about 3 times larger (same size). This is a consequence of the different bending angles at $\mathrm{LHCb}$ and $\mathrm{S}_{2}$, i.e. 16 and $7 \mathrm{mrad}$, respectively. For germanium at $77 \mathrm{~K}$, with yields higher by a factor of 3 (2) and momentum spectra similar to germanium $S_{2}$, the uncertainties are reduced by an equivalent data sample close to a factor of 4 (3) larger. Silicon provides significantly lower yields and softer momentum spectra, resulting in sensitivities whose equivalent data sample size is a factor of 4 (3) smaller. EDM sensitivities strongly depend on the anomalous magnetic moment $a$, although they present similar features. Thus, improved sensitivities with respect to the LHCb detector based configurations are expected at $\mathrm{S}_{2}$ if much higher PoT would be achievable.

\section{CONCLUSIONS}

In summary, progress towards the first measurement of charm baryons dipole moments is reported. An experimental setup based on bent crystals and a W target placed upstream of the $\mathrm{LHCb}$ detector is studied. Silicon and germanium long bent crystals have been tested on a $180 \mathrm{GeV}$ hadron beam with relatively high channeling efficiency measured for both prototypes. The germanium crystal provides enhanced sensitivity to MDM and EDM compared to the silicon crystal, especially when cooled down at $77 \mathrm{~K}$ [19]. Advanced analysis techniques have been developed for three- and four-body charm baryon decays, providing enhanced sensitivity to the measurements. For a baseline configuration with $1.37 \times 10^{13} \mathrm{PoT}$ impinging on $2 \mathrm{~cm} \mathrm{~W}$ target and with a germanium crystal at room temperature, similar sensitivities for the MDM $(\mathrm{EDM})$ of $\Lambda_{c}^{+}$and $\Xi_{c}^{+}$baryons below $2 \times 10^{-2} \boldsymbol{\mu}_{\mathrm{N}}$ $\left(3 \times 10^{-16} e \mathrm{~cm}\right)$ are achievable, which corresponds to a relative precision on the gyromagnetic factors $g$ of about $4 \%$. A germanium crystal cooled at $77 \mathrm{~K}$ would improve the sensitivity by a factor of 2 . These results enable a unique program of MDM and EDM measurements of charm baryons at $\mathrm{LHCb}$, capable to test advanced low energy models of strong interactions and to search for 
physics beyond the SM. A future dedicated experiment with significantly higher PoT would offer the possibility to improve the sensitivity to charm baryon dipole moments, and to explore beauty baryons and ultimately the $\tau$ lepton [54,62].

\section{ACKNOWLEDGMENTS}

We express our gratitude to our colleagues of the $\mathrm{LHCb}$ collaboration in particular to M. Ferro-Luzzi, M. Palutan, C. Parkes, P. Robbe, N. Tuning, and from CERN, in particular A. Fomin, D. Mirarchi, S. Redaelli for stimulating discussions and very useful feedback. We would like to thank S. Forte and J. Rojo for useful discussions on parton distribution functions. A. Sytov thanks V. Haurylavets and A. Leukovich for providing help with GEANT4 simulations. We acknowledge support from INFN (Italy), MICINN and GVA (Spain), the ERC Consolidator Grants SELDOM G. A. 771642 and CRYSBEAM G. A. 615089. In addition, we acknowledge the CINECA award under the ISCRA initiative for the availability of high performance computing resources and support.

\section{APPENDIX A: MANUFACTURING OF CRYSTAL AND ITS BENDER}

Silicon and germanium crystals are prepared starting from wafers available from commercial suppliers. The thickness of the wafers is 5 and $1 \mathrm{~mm}$ for the silicon and germanium wafers, respectively. Wafers with a dislocation density lower than $1 / \mathrm{cm}^{2}$ over the entire region interacting with the particle beam are selected from a stock of wafers. The density of dislocations is characterized through the etch pit density $[63,64]$ and the x-ray topography techniques. The miscut angle, i.e. the angle between the optical surface of the wafer surface and the atomic planes, is measured using a high-resolution X-ray diffractometer (Panalytical X'Pert ${ }^{3}$ MRD XL) coupled to an autocollimator. Subsequently, the miscut is reduced to less than $0.01^{\circ}$ through polishing with a Logitech PM5 equipment. The wafers are diced with a dicing machine (Disco DAD3220) to rectangular crystals, then cleaned in a hot bath of acetone under ultrasonic agitation. Bending of the crystal occurs as a consequence of clamping of the crystal between surfaces of a properly machined bender. Assemblies describing crystal benders are modeled through finite element modeling (Ansys R18), and the shape of the surface in contact with the crystal are properly modeled to maximize uniformity of the deformation of the crystal. Benders are manufactured through milling and electrodischarge machining of a block of stainless steel 316LN. This material is chosen for its compatibility with the environment of LHC, where the devices are supposed to operate. After machining, the bender is cleaned in an acetone bath under ultrasonic agitation. To avoid interference of dust which might deposit on the surfaces of the bender or of the crystal, the assembly of crystal on the bender is accomplished in a ISO-4 clean room.

\section{APPENDIX B: SIMULATION STUDIES}

\section{Simulation of interaction between particle beam and crystal}

A precise treatment of scattering of channeled particles in the crystal is fundamental for the design of experiments aiming to study spin precession of short lifetime particles. Scattering from nuclei and inner shell electrons plays an important role in describing the dynamics of channeled particles, rendering the description of this process more difficult. Propagation of particles in the crystal is described by the Monte Carlo simulation CRYSTALRAD [30,65]. State of the art simulations are based on the solution of the equation of motion of a charged particle interacting with a crystalline lattice and are quantum-mechanically grounded, including refined treatments of both large- and small-angle scattering. Recently observed experimentally [66] incoherent scattering modification is also part of modern simulation codes. Bent crystals cooled to cryogenic temperatures are more efficient than at room temperature due to the lower vibration amplitude of the atoms [67]. According to channeling simulations the improvement is more significant for germanium than for silicon crystals. Steering efficiencies for different crystal configurations considered in the sensitivity studies, as obtained from these simulations, are shown in Fig. 9.

\section{Evaluation of the $\Lambda_{c}^{+}$and $\Xi_{c}^{+}$spectra after the tungsten target and the crystal}

The variation per unit length of the number of protons, $N_{p}$, due to the interaction with the tungsten target is given by

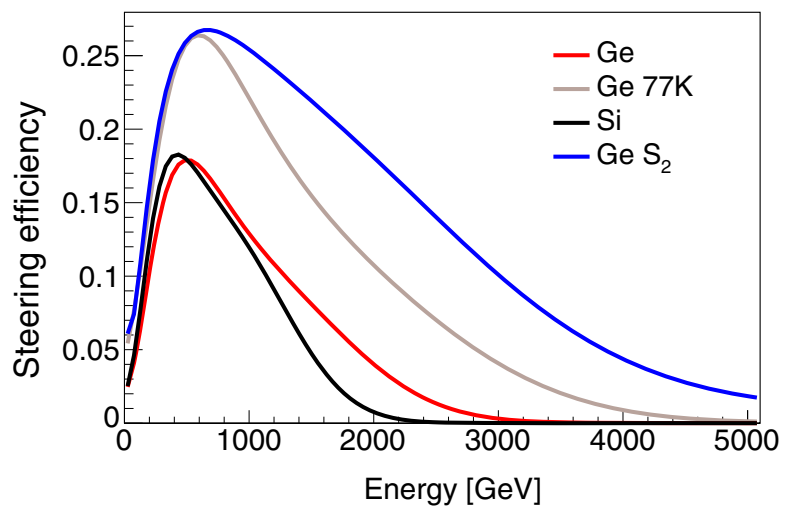

FIG. 9. Monte Carlo simulation results for the steering efficiency vs energy of particles impinging parallel to the atomic planes, assuming uniform bending radius, of germanium and silicon crystals with deflection angle $16 \mathrm{mrad}$ and $10 \mathrm{~cm}$ length (LHCb scenario), and germanium $7 \mathrm{mrad}$ bent and $7 \mathrm{~cm}$ length $\left(S_{2}\right)$, at room temperature. For germanium in the LHCb scenario results with cooling at $77 \mathrm{~K}$ are also shown. 


$$
\frac{\mathrm{d} N_{p}}{\mathrm{~d} z}=-\frac{N_{p}}{\lambda_{\mathrm{W}}}
$$

where $\lambda_{\mathrm{W}}=A_{T} /\left(\rho N_{A} A_{\mathrm{W}}^{\text {part }} \sigma_{p N}\right) \approx 8.87 \mathrm{~cm}$ is the $\mathrm{W}$ nuclear interaction length at $\sqrt{s} \approx 115 \mathrm{GeV}$, with $\rho$ and $A_{T}$ the W density and atomic mass respectively, $N_{A}$ the Avogadro number, $A_{\mathrm{W}}^{\mathrm{p} \text { part }}$ the number of participant nucleons calculated on the basis of the Glauber model $[68,69]$, and $\sigma_{p N}$ the proton-nucleon inelastic cross section at $\sqrt{s} \approx 115 \mathrm{GeV}$ [47].

The variation per unit length of the number of $\Lambda_{c}^{+}$(and similarly $\Xi_{c}^{+}$) baryons $N_{\Lambda_{c}^{+}}$, is determined by the disappearance of protons in the $p \mathrm{~W}$ interaction, and by the decay and nuclear interaction in the target of the produced $\Lambda_{c}^{+}$baryons,

$$
\frac{\mathrm{d} N_{\Lambda_{c}^{+}}}{\mathrm{d} z}=\frac{N_{\mathrm{PoT}}}{\lambda_{\mathrm{W}, \Lambda_{c}^{+}}} e^{-z / \lambda_{\mathrm{W}}}-\frac{N_{\Lambda_{c}^{+}}}{\lambda^{\prime}},
$$

where $1 / \lambda^{\prime}=1 / \lambda_{\mathrm{W}}^{\left(\Lambda_{c}^{+}\right)}+1 /(\beta \gamma c \tau), \beta \gamma$ is the $\Lambda_{c}^{+}$Lorentz boost factor, $\tau$ its lifetime, $c$ the speed of light, $N_{\mathrm{PoT}}$ the number of protons hitting the target, $\lambda_{\mathrm{W}}^{\left(\Lambda_{c}^{+}\right)} \approx \lambda_{\mathrm{W}}$ the $\Lambda_{c}^{+}$interaction length, and $\lambda_{\mathrm{W}, \Lambda_{c}^{+}}$is the mean-free path for $\Lambda_{c}^{+}$production. The latter is estimated as $\lambda_{\mathrm{W}, \Lambda_{c}^{+}}=$ $A_{T} /\left(\rho N_{A} A_{N} \sigma_{\Lambda_{c}^{+}}\right) \approx 81.35 \mathrm{~m}$, where $A_{N}$ is the $\mathrm{W}$ atomic mass number and $\sigma_{\Lambda_{c}^{+}}$the proton nucleon to $\Lambda_{c}^{+}$cross section at $\sqrt{s} \approx 115 \mathrm{GeV}$, obtained by scaling linearly the measured proton nucleon to $c \bar{c}$ cross section at $\sqrt{s}=$ $86.6 \mathrm{GeV}$ [70] and using the $\Lambda_{c}^{+}$fragmentation fraction [71,72]. For $\Xi_{c}^{+}$baryons the fragmentation fraction is estimated to be about 0.7 times smaller than for $\Lambda_{c}^{+}$, assuming it to be similar for $\Xi_{c}^{+}, \Xi_{c}^{0}$ and $\Omega_{c}^{0}$ baryons [18], leading to $\lambda_{\mathrm{W}, \Xi_{c}^{+}} \approx 114.72 \mathrm{~m}$. Integration of Eq. (B2) for a target of thickness $z$ and fixed $\beta \gamma$ yields

$$
N_{\Lambda_{c}^{+}}(z, \beta \gamma)=\frac{N_{\mathrm{PoT}}}{\lambda_{\mathrm{W}, \Lambda_{c}^{+}}} \beta \gamma c \tau\left(e^{-z / \lambda_{\mathrm{W}}}-e^{-z / \lambda^{\prime}}\right) .
$$

The number of $\Lambda_{c}^{+}$baryons after traversing a thickness $z$ of the target, $N_{\Lambda_{c}^{+}}(z)$, is then given by the convolution of Eq. (B3) with the normalized $\Lambda_{c}^{+}$baryon momentum spectra, $\quad r(\beta \gamma)=1 / \sigma_{\Lambda_{c}^{+}} \mathrm{d} \sigma_{\Lambda_{c}^{+}}(\boldsymbol{p}) / \mathrm{d}^{3} \boldsymbol{p}$, estimated using Monte Carlo simulations based on PYTHIA. Figure 10 shows the number of $\Lambda_{c}^{+}$and $\Xi_{c}^{+}$baryons exiting the target as a function of the target thickness for the case of $N_{\mathrm{PoT}}=$ $1.37 \times 10^{13}$ protons.

The number of $\Lambda_{c}^{+}$baryons produced in a target of thickness $T$ and traversing a bent crystal of length $L$ is given by the convolution of

$N_{\Lambda_{c}^{+}, \text {cry }}(T, L, \beta \gamma)=N_{\Lambda_{c}^{+}}(T, \beta \gamma) \epsilon_{\mathrm{CH}}(\beta \gamma) \epsilon_{\mathrm{DF}}(L, \beta \gamma)$

with $r(\beta \gamma)$, where $\epsilon_{\mathrm{CH}}(\beta \gamma)$ is the steering efficiency of the crystal, which includes the efficiency of the $\Lambda_{c}^{+}$

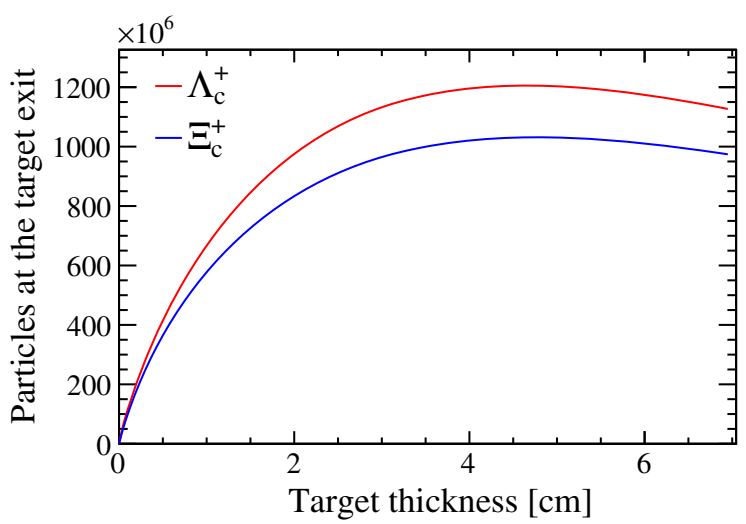

FIG. 10. Number of produced $\Lambda_{c}^{+}$and $\Xi_{c}^{+}$baryons exiting the $\mathrm{W}$ target as a function of the target thickness. The case of $1.37 \times 10^{13}$ PoT, corresponding to two years running in $\mathrm{LHCb}$, is shown.

particle to be trapped into channeling regime, and $\epsilon_{\mathrm{DF}}(T, L, \beta \gamma)=e^{-L / \beta \gamma c \tau}$ accounts for the particle decay flight, i.e. survival probability within the crystal length.

\section{Reconstruction of $\Lambda_{c}^{+}$and $\Xi_{c}^{+}$decays with a $\pi^{0}$ in the final state}

The momentum in the laboratory frame of a $\Lambda_{c}^{+}$(and similarly for $\Xi_{c}^{+}$) baryon decaying into final states containing an undetected neutral pion can be reconstructed, up to a twofold ambiguity, using kinematic information of the remaining charged-particle decay products $(3 h)$. The ambiguity arises from two different configurations in the baryon rest frame which are indistinguishable in the laboratory frame. Either a relatively low momentum $\Lambda_{c}^{+}$charm baryon decays into the $3 h$ system with a small angle with respect to the $\Lambda_{c}^{+}$flight direction, or the $3 h$ system is produced with a large angle from a higher momentum $\Lambda_{c}^{+}$. The ambiguity vanishes for events in which the angle $\theta$ between the flight direction of the baryon and the $3 h$ system reaches its maximum allowed value,

$$
\theta_{\max }=\arcsin \frac{\sqrt{\left(m^{2}-m_{3 h}^{2}-m_{\pi^{0}}^{2}\right)^{2}-4 m_{3 h}^{2} m_{\pi^{0}}^{2}}}{2 m p_{3 h}},
$$

leading to a single solution

$$
p=\frac{p_{3 h}\left(m^{2}+m_{3 h}^{2}-m_{\pi^{0}}^{2}\right) \cos \theta_{\max }}{2\left(m_{3 h}^{2}+p_{3 h}^{2} \sin ^{2} \theta_{\max }\right)},
$$

which is used as an estimate of the $\Lambda_{c}^{+}$momentum magnitude. Here, $p_{3 h}$ and $m_{3 h}$ are the momentum magnitude and invariant mass of the $3 h$ system, respectively, $m$ is the nominal charm baryon mass, and $m_{\pi^{0}}$ the neutral pion mass. This procedure does not impose the additional kinematical constraint when the $\pi^{0}$ meson is produced via $\Sigma^{+}$decays. The $\Lambda_{c}^{+}$three-momentum then can be determined from the $\Lambda_{c}^{+}$flight direction, itself obtained from the 


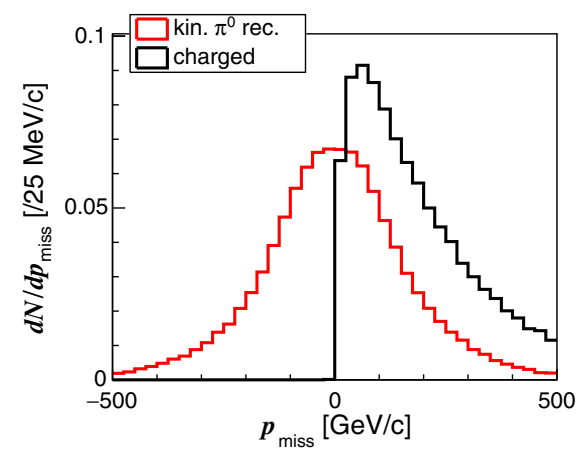

(a)

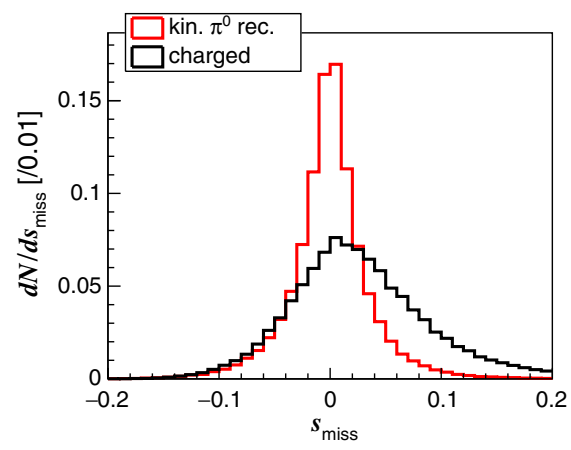

(b)

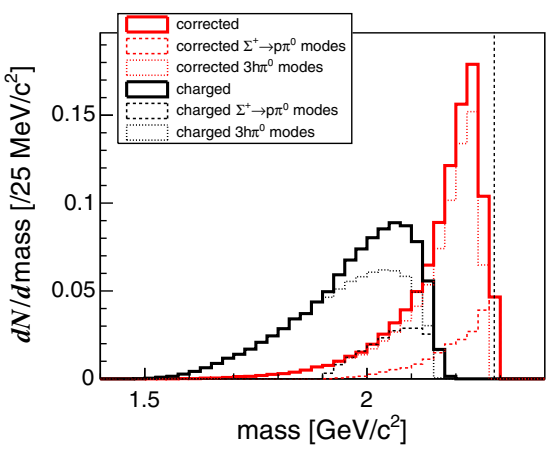

(c)

FIG. 11. Distributions of missing (a) momentum and (b) spin-polarization magnitudes using the kinematical reconstruction technique for $\Lambda_{c}^{+}$decays with an undetected neutral pion (red) in the final states listed in Table II. As a comparison, the missing momentum and polarization magnitudes for the reconstructed $3 h$ system (black), composed by a proton combined with charged kaons and pions, are shown. The relative contributions from the different final states are given by the effective branching fractions $\mathcal{B}_{\text {eff }}$. (c) Corrected mass distribution for the same decays. As a comparison the invariant mass of the reconstructed charged $3 h$ system, composed by a proton or a $\Sigma^{ \pm}$hyperon combined with charged kaons and pions, is shown. The $\Lambda_{c}^{+}$known mass is reported as a vertical dashed line. The estimated invariant mass resolution at $\mathrm{LHCb}$, about $15 \mathrm{MeV} / c^{2}$, is negligible in comparison to the width of the corrected mass distribution. All distributions are normalized to unity.

knowledge of the production (primary), $\boldsymbol{x}_{\mathrm{PV}}$, and the $\Lambda_{c}^{+}$ decay, $\boldsymbol{x}_{\Lambda_{c}^{+}}$, vertex positions, as $\boldsymbol{p}=p \boldsymbol{u}$, where $\boldsymbol{u}$ is a unit vector along the $\Lambda_{c}^{+}$flight direction,

$$
\boldsymbol{u}=\frac{\boldsymbol{x}_{\Lambda_{c}^{+}}-\boldsymbol{x}_{\mathrm{PV}}}{\left|\boldsymbol{x}_{\Lambda_{c}^{+}}-\boldsymbol{x}_{\mathrm{PV}}\right|}
$$

The average initial spin-polarization vector can then be inferred from Eq. (8).

Figures 11(a) and 11(b) illustrate the difference between the estimated momentum and spin-polarization magnitudes using the kinematical recovery technique and the true $\Lambda_{c}^{+}$ momentum, compared to the case when the $\Lambda_{c}^{+}$threemomentum and polarization vectors are estimated using the $3 h$ system only. Whereas the former provides unbiased estimates with resolutions about $150 \mathrm{GeV} / c^{2}$ and 0.05 , respectively, the latter is clearly biased. Estimated vertex resolutions around $100 \mu \mathrm{m}$ and $10 \mathrm{~mm}$ in the transverse and longitudinal directions, respectively, along with an invariant mass resolution of about $15 \mathrm{MeV} / c^{2}[18]$, do not impact significantly these distributions.

To characterize efficiently and with low background signal $\Lambda_{c}^{+}$decays with undetected neutral pions, the corrected mass [53], defined as

$$
m_{\mathrm{corr}}=\sqrt{m_{3 h}^{2}+p_{\perp}^{2}}+p_{\perp},
$$

is used, where $m_{3 h}$ is the reconstructed invariant mass of the three charged tracks in the final state and $p_{\perp}$ is the momentum of the $3 h$ system transverse to the charm baryon flight direction, estimated as

$$
p_{\perp}=\left|\boldsymbol{p}_{3 h} \times \boldsymbol{u}\right| .
$$

Figure 11(c) shows the $m_{\text {corr }}$ distribution for the mixture of three- and four-body $\Lambda_{c}^{+}$decay modes listed in Table II with a $\pi^{0}$ in the final state, and compared with the invariant mass of the $3 h$ system and the known $\Lambda_{c}^{+}$baryon mass [47].

\section{Sensitivity studies and crystal setup optimization}

Sensitivity studies for the MDM and EDM of $\Lambda_{c}^{+}$and $\Xi_{c}^{+}$ baryons are based on PYTHIA simulations and pseudoexperiments where the number of events is estimated according to the branching fractions reported in Tables II and III, respectively. The values for the production cross sections, the average event information $S^{2}$, and the gyromagnetic factors are reported in Table IV. Other relevant parameters used for the sensitivity studies, also reported, are evaluated for each different setup configuration according to simulations: in particular, the channeling efficiency $\epsilon_{\mathrm{CH}}$, the decay flight efficiency $\epsilon_{\mathrm{DF}}$, the number of recontructed charm baryons $N_{\text {rec }}$, the average boost $\langle\gamma\rangle$ and transverse momentum $\left\langle p_{\mathrm{T}}\right\rangle$, and the initial average spin-polarization vector $\boldsymbol{s}$. For the pseudoexperiments, Eq. (10) is used for spin-polarization projections after precession in the crystal.

Regions of minimal uncertainty of $d$ and $g$ factors are explored for different crystal configurations and target thickness, with deflection angles of 16 and $7 \mathrm{mrad}$. These steering angles are chosen for compatibility with the $\mathrm{LHCb}$ detector acceptance and to allow effective separation of channeled $\Lambda_{c}^{+}$baryons from forwardly produced background particles in the $S_{2}$ scenario [18], respectively. Crystal lengths around 10 and $7 \mathrm{~cm}$ are chosen to minimize material for detector operation and safety while accepting a $50 \%$ and below $20 \%$ increase of uncertainties, respectively. The crystal orientation angle and target thickness are chosen to be $0.3 \mathrm{mrad}$ and $2 \mathrm{~cm}$, respectively. 
To assess the benefits of using event-by-event information, Fig. 12(a) shows sensitivity regions as a function of the crystal orientation angle and the target thickness for combined MDM and EDM measurement, using average event information. These regions and minimal uncertainties are to be compared to those illustrated in Fig. 5(b). A sensitivity improvement equivalent to a data sample size larger than a factor of 3 (of 2 for MDM measurement alone) is observed when using event-by-event estimates of the precession angle $\Phi$ and the initial polarizationvector $s$ with respect to the case when these are averaged over all events.

First order, analytical approximation to the dipole moment sensitivities is given by

$$
\begin{aligned}
\sigma_{g} & \approx \frac{2}{S\left\langle s_{y}\right\rangle\langle\gamma\rangle \theta_{C}} \frac{1}{\sqrt{N_{\text {rec }}}}, \\
\sigma_{d} & \approx \frac{g-2}{S} \frac{1}{\sqrt{\left\langle s_{y}\right\rangle^{2} \eta^{2}+2\left\langle s_{x}\right\rangle^{2} \eta}} \frac{1}{\sqrt{N_{\text {rec }}}},
\end{aligned}
$$

with $\eta=1-\cos \langle\Phi\rangle$, which follows from Eq. (10) in the limit $a \gg d, 1 / \gamma$,

$$
\begin{aligned}
& s_{x}^{\prime} \approx s_{y} \frac{d}{g-2}(1-\cos \Phi)+s_{x}, \\
& s_{y}^{\prime} \approx s_{y} \cos \Phi+s_{x} \frac{d}{g-2}(1-\cos \Phi), \\
& s_{z}^{\prime} \approx-s_{y} \sin \Phi+s_{x} \frac{d}{g-2} \sin \Phi,
\end{aligned}
$$

where $\Phi$ is given by Eq. (1). In this case the uncertainties are estimated separately for events with positive and negative $p_{x_{L}}$, and then combined. Figure 12(b) shows the corresponding sensitivity regions. Differences with respect to Fig. 12(a) are related to the approximations in Eq. (B11) and the correlations between $g$ and $d$ in the combined measurement, neglected in the analytical estimates. The dependence of the EDM sensitivity with the crystal orientation angle is more evident in Fig. 12(b) due to an incomplete and approximated use of the spin precession information.

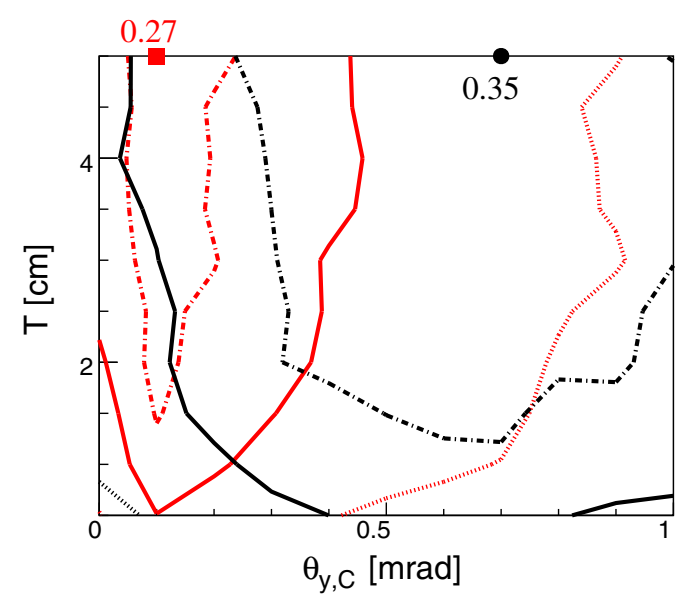

(a)

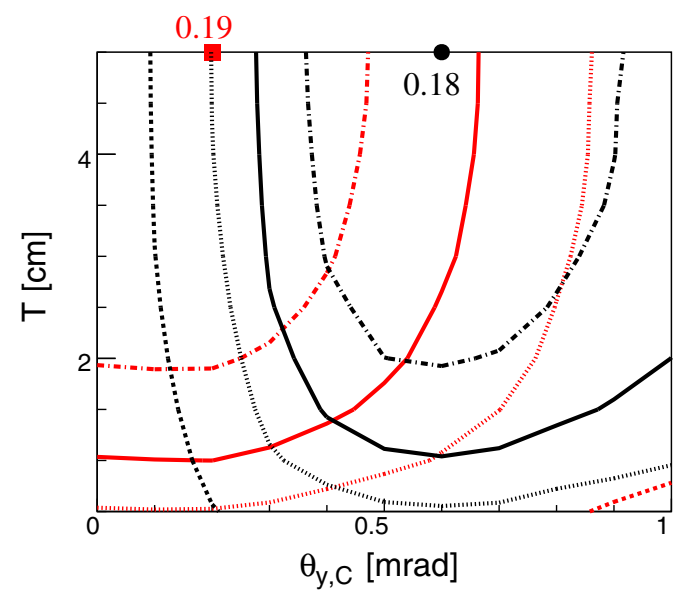

(b)

FIG. 12. Regions of minimal uncertainty of the $g$ (red curves) and $d$ (black) factors as a function of the crystal orientation angle $\theta_{y, C}$ and the target thickness $T$, with $a \approx-0.03$ and $\Lambda_{c}^{+} \rightarrow p K^{-} \pi^{+}$ decays, (a) for combined MDM and EDM measurement using average estimates of the precession angle $\Phi$ and the initial polarization-vector $s$, and (b) from first order, analytical estimates as given by Eq. (B10). The markers and values represent the minimum uncertainty on the $g$ and $d$ factors, relative to $1.37 \times 10^{13}$ PoT and a $16 \mathrm{mrad}$ bent, $10 \mathrm{~cm}$ long germanium crystal at room temperature. The curves are the regions whose uncertainties are increased by $20 \%, 50 \%$ and $100 \%$ with respect to the minimum.
[1] G. Schneider, A. Mooser, M. Bohman, N. Schön, J. Harrington, T. Higuchi, H. Nagahama, S. Sellner, C. Smorra, K. Blaum et al., Science 358, 1081 (2017).

[2] B. K. Sahoo, Phys. Rev. D 95, 013002 (2017).

[3] J. M. Pendlebury, S. Afach, N. J. Ayres, C. A. Baker, G. Ban, G. Bison, K. Bodek, M. Burghoff, P. Geltenbort, K. Green et al., Phys. Rev. D 92, 092003 (2015).
[4] G. W. Bennett et al. (Muon $g-2$ Collaboration), Phys. Rev. D 73, 072003 (2006).

[5] G. W. Bennett et al. (Muon $g-2$ Collaboration), Phys. Rev. D 80, 052008 (2009).

[6] D. Hanneke, S. Fogwell, and G. Gabrielse, Phys. Rev. Lett. 100, 120801 (2008).

[7] J. Baron et al. (ACME Collaboration), Science 343, 269 (2014). 
[8] A. Dainese et al. (QCD Working Group), arXiv:1901 .04482.

[9] J. P. Miller, E. de Rafael, and B. L. Roberts, Rep. Prog. Phys. 70, 795 (2007).

[10] T. Chupp, P. Fierlinger, M. Ramsey-Musolf, and J. Singh, Rev. Mod. Phys. 91, 015001 (2019).

[11] J. Beacham et al., J. Phys. G 47, 010501 (2020).

[12] Y. Ünal and U.-G. Meißner, J. High Energy Phys. 01 (2021) 115.

[13] F. Sala, J. High Energy Phys. 03 (2014) 061.

[14] G. Hector and J. Ruiz Vidal, Phys. Rev. D 101, 115010 (2019).

[15] V. G. Baryshevsky, Phys. Lett. B 757, 426 (2016).

[16] L. Burmistrov et al. (UA9 Collaboration), CERN Reports No. CERN-SPSC-2016-030 and No. SPSC-EOI-012, 2016.

[17] F. J. Botella, L. M. Garcia Martin, D. Marangotto, F. Martinez Vidal, A. Merli, N. Neri, A. Oyanguren, and J. Ruiz Vidal, Eur. Phys. J. C 77, 181 (2017).

[18] E. Bagli, L. Bandiera, G. Cavoto, V. Guidi, L. Henry, D. Marangotto, F. Martinez Vidal, A. Mazzolari, A. Merli, N. Neri et al., Eur. Phys. J. C 77, 828 (2017); 80, 680(E) (2020).

[19] A. S. Fomin, A. Y. Korchin, A. Stocchi, O. A. Bezshyyko, L. Burmistrov, S. P. Fomin, I. V. Kirillin, L. Massacrier, A. Natochii, P. Robbe et al., J. High Energy Phys. 08 (2017) 120 .

[20] D. Chen et al. (E761 Collaboration), Phys. Rev. Lett. 69, 3286 (1992).

[21] V. Samsonov, Nucl. Instrum. Meth. B 119, 271 (1996).

[22] V. V. Baublis, A. V. Khanzadeev, V. M. Samsonov, N. K. Terentev, A. A. Vorobev, R. A. Carrigan. Jr., D. Chen, P. S. Cooper, D. C. Daniels, J. Lach et al., Nucl. Instrum. Meth. B 90, 112 (1994).

[23] A. Khanzadeev, V. Samsonov, R. A. Carrigan, and D. Chen, Nucl. Instrum. Methods Phys. Res., Sect. B 119, 266 (1996).

[24] V. G. Baryshevsky, Pisma Zh. Tekh. Fiz. 5, 182 (1979).

[25] J. Lindhard and K. Dan, Vidensk. Selsk. Mat. Fys. Medd. 34, 14 (1965).

[26] V. M. Biryukov, Y. A. Chesnokov, and V. I. Kotov, Crystal Channeling and Its Application at High-Energy Accelerators (Springer-Verlag, Berlin, Heidelberg, 1997).

[27] R. Aaij et al. (LHCb Collaboration), Int. J. Mod. Phys. A 30, 1530022 (2015).

[28] I. Bediaga et al. (LHCb Collaboration), CERN Reports No. CERN-LHCC-2012-007 and No. LHCb-TDR-12, 2012.

[29] A. S. Fomin, S. Barsuk, A. Y. Korchin, V. A. Kovalchuk, E. Kou, M. Liul, A. Natochii, E. Niel, P. Robbe, and A. Stocchi, Eur. Phys. J. C 80, 358 (2020).

[30] A. I. Sytov and V. V. Tikhomirov, Nucl. Instrum. Methods Phys. Res., Sect. B 355, 383 (2015).

[31] E. Bagli, V. Guidi, A. Mazzolari, L. Bandiera, G. Germogli, A. I. Sytov, D. De Salvador, A. Argiolas, M. Bazzan, A. Carnera et al., Phys. Rev. Lett. 115, 015503 (2015).

[32] S. Baricordi, V. Guidi, A. Mazzolari, G. Martinelli, A. Carnera, D. De Salvador, A. Sambo, G. Della Mea, R. Milan, A. Vomiero et al., Appl. Phys. Lett. 91, 061908 (2007).
[33] G. Germogli, A. Mazzolari, L. Bandiera, E. Bagli, and V. Guidi, Nucl. Instrum. Methods Phys. Res., Sect. B 355, 81 (2015).

[34] A. Mazzolari, M. Romagnoni, R. Camattari, E. Bagli, L. Bandiera, G. Germogli, V. Guidi, and G. Cavoto, Eur. Phys. J. C 78, 720 (2018).

[35] W. Scandale, G. Arduini, M. Butcher, F. Cerutti, M. Garattini, S. Gilardoni, A. Lechner, R. Losito, A. Masi, D. Mirarchi et al., Phys. Lett. B 758, 129 (2016).

[36] G. Germogli, A. Mazzolari, V. Guidi, and M. Romagnoni, Nucl. Instrum. Meth. B 402, 308 (2017).

[37] S. Agostinelli et al. (GEANT4 collaboration), Nucl. Instrum. Meth. A 506, 250 (2003).

[38] A. Taratin and S. Vorobiev, Phys. Lett. A 119, 425 (1987).

[39] W. Scandale, G. Arduini, M. Butcher, F. Cerutti, M. Garattini, S. Gilardoni, L. Lari, A. Lechner, R. Losito, A. Masi et al., Phys. Lett. B 743, 440 (2015).

[40] R. Rossi, G. Cavoto, D. Mirarchi, S. Redaelli, and W. Scandale, Nucl. Instrum. Meth. B 355, 369 (2015).

[41] T. Sjöstrand, S. Ask, J. R. Christiansen, R. Corke, N. Desai, P. Ilten, S. Mrenna, S. Prestel, C. O. Rasmussen, and P. Z. Skands, Comput. Phys. Commun. 191, 159 (2015).

[42] R. D. Ball et al. (NNPDF Collaboration), Eur. Phys. J. C 77, 663 (2017).

[43] V. Bertone et al. (NNPDF Collaboration), SciPost Phys. 5, 008 (2018).

[44] V. Bertone, R. Gauld, and J. Rojo, J. High Energy Phys. 01 (2019) 217, and references therein.

[45] M. Jacob and G. Wick, Ann. Phys. (N.Y.) 7, 404 (1959).

[46] M. Davier, L. Duflot, F. Le Diberder, and A. Rouge, Phys. Lett. B 306, 411 (1993).

[47] M. Tanabashi et al. (Particle Data Group), Phys. Rev. D 98, 030001 (2018).

[48] S. Barlag et al. (ACCMOR Collaboration), Phys. Lett. B 325, 531 (1994).

[49] E. M. Aitala et al. (E791 Collaboration), Phys. Lett. B 471, 449 (2000).

[50] D. Marangotto, Amplitude analysis and polarization measurement of the $\Lambda_{c}^{+}$baryon in $p K^{-} \pi^{+}$final state for electromagnetic dipole moment experiment, $\mathrm{Ph} . \mathrm{D}$. thesis, Milan University, 2020.

[51] D. Marangotto, Adv. High Energy Phys. 2020, 7463073 (2020).

[52] Y. B. Li et al. (Belle Collaboration), Phys. Rev. D 100, 031101 (2019).

[53] K. Abe et al. (SLD Collaboration), Phys. Rev. Lett. 80, 660 (1998).

[54] J. Fu, M. A. Giorgi, L. Henry, D. Marangotto, F. M. Vidal, A. Merli, N. Neri, and J. Ruiz Vidal, Phys. Rev. Lett. 123, 011801 (2019).

[55] R. Aaij et al. (LHCb Collaboration), J. Instrum. 9, P09007 (2014).

[56] A. Bursche, H. P. Dembinski, P. Di Nezza, M. Ferro-Luzzi, F. Fleuret, G. Graziani, G. Manca, E. A. Maurice, N. Neri, L. L. Pappalardo et al., CERN Reports No. LHCb-PUB2018-015 and No. CERN-LHCb-PUB-2018-015, 2018.

[57] L. H. Thomas, Nature (London) 117, 514 (1926).

[58] L. H. Thomas, Philos. Mag. 3, 1 (1927). 
[59] V. Bargmann, L. Michel, and V. L. Telegdi, Phys. Rev. Lett. 2, 435 (1959).

[60] D. Mirarchi, A. Fomin, S. Redaelli, and W. Scandale, Eur. Phys. J. C 80, 929 (2020).

[61] V. G. Baryshevsky, Phys. Rev. Accel. Beams 22, 081004 (2019).

[62] A. S. Fomin, A. Y. Korchin, A. Stocchi, S. Barsuk, and P. Robbe, J. High Energy Phys. 03 (2019) 156.

[63] E. Sirtl and A. Adler, Z. Metallkd. 52, 529 (1961).

[64] F. S. d. Aragona, J. Electrochem. Soc. 119, 948 (1972).

[65] A. I. Sytov, V. V. Tikhomirov, and L. Bandiera, Phys. Rev. Accel. Beams 22, 064601 (2019).

[66] A. Mazzolari, A. Sytov, L. Bandiera, G. Germogli, M. Romagnoni, E. Bagli, V. Guidi, V. V. Tikhomirov,
D. De Salvador, S. Carturan et al., Eur. Phys. J. C 80, 63 (2020).

[67] J. S. Forster, H. Hatton, R. J. Toone, G. Este, S. I. Baker, R. A. Carrigan, W. M. Gibson, R. L. Wijayawardana, J. A. Ellison, L. Emman-Wori et al., Nucl. Phys. B318, 301 (1989).

[68] M. L. Miller, K. Reygers, S. J. Sanders, and P. Steinberg, Annu. Rev. Nucl. Part. Sci. 57, 205 (2007).

[69] C. Loizides, Phys. Rev. C 94, 024914 (2016).

[70] R. Aaij et al. (LHCb Collaboration), Phys. Rev. Lett. 122, 132002 (2019).

[71] M. Lisovyi, A. Verbytskyi, and O. Zenaiev, Eur. Phys. J. C 76, 397 (2016).

[72] L. Gladilin, Eur. Phys. J. C 75, 19 (2015). 